\title{
Representation Varieties and Character Varieties
}

By

\author{
Kazunori NAKAMOTO*
}

The aim of this paper is to construct a coarse moduli scheme over $\mathbf{Z}$ of equivalence classes of absolutely irreducible representations of each degree of an arbitrary group (Theorem 1.3). Roughly speaking, we attach a Z Z-scheme structure on the set of equivalence classes of absolutely irreducible representations. We call this moduli scheme the "character variety". Similarly we can construct the character varieties for absolutely irreducible representations of semigroups, monoids, associative algebras, and Lie algebras (Remarks 1.4 and 1.5).

The moduli spaces of representations have been used for describing the moduli spaces of various geometric objects. For example, the moduli of stable vector bundles over a compact Riemann surface of genus $\geq 2$ is described as the set of equivalence classes of irreducible unitary representations of the fundamental group. Another example is the Fricke space, that is, the Teichmüller space of Riemann surfaces, which is described as the set of equivalence classes of irreducible representations of the fundamental group in $\mathrm{PSL}_{2}(\mathbf{R})$ or $\mathrm{SL}_{2}(\mathbf{R})$. In this way, there are many applications of the character variety from a geometric viewpoint. However in this article we will forget original geometric motivations. Our subject is to establish a general framework for describing the representations of an arbitrary group apart from a geometric viewpoint.

In this paper the discussion is all done over $\mathbf{Z}$, and we deal with representations over an arbitrary commutative ring. We introduce the notion of absolute irreducibility of representations over any commutative ring (Definition 1.1). We also extend the notion of equivalence relation of representations (Definition 1.2). We remark that there is no condition on groups for constructing the character varieties. In the process of the construction we obtain the following result on the representation theory: for any two absolutely irreducible representations $\rho, \rho^{\prime}: \Gamma \rightarrow \mathrm{GL}_{n}(R)$ with a group $\Gamma$ and a commutative

Communicated by Y. Ihara, July 4, 1997. Revised July 10, 1998 and October 21, 1999.

1991 Mathematics Subject Classification(s): 14D22 (20C99, 16G99, 14D20, 11F80)

* Department of Mathematics, Faculty of Science, Kyoto University, Kyoto 606-8502, Japan.

e-mail: nakamoto@kusm.kyoto-u.ac.jp 
ring $R$, they are equivalent if and only if they have the same traces (Theorem 6.12). This theorem, which strengthens the results of Carayol [1] and Serre [10], implies one of the universal properties of representations independent of groups and coefficient commutative rings. It is essential for this theorem that the subalgebra $R[\rho(\Gamma)]$ generated by the image $\rho(\Gamma)$ coincides with the full matrix ring $\mathrm{M}_{n}(R)$, that is, $\rho$ is absolutely irreducible. We succeed in describing equivalence classes of representations by their invariants (traces, determinants and so on) in the absolutely irreducible case. Investigating absolutely irreducible representations for each group can be reduced to studying the character varieties of each group. In this article we do not go into the detail on the case that representations are not absolutely irreducible; we leave that case until the following papers.

To construct the character variety, we need to introduce the "representation variety". The representation variety of degree $n$ for a group $\Gamma$ is the affine scheme $\operatorname{Rep}_{n}(\Gamma)$ parameterizing the representations of degree $n$ for $\Gamma$. The group scheme $\operatorname{PGL}_{n}$ over $\mathbb{Z}$ acts on $\operatorname{Rep}_{n}(\Gamma)$ by $\rho \mapsto P^{-1} \rho P$, where $\rho \in \operatorname{Rep}_{n}(\Gamma)$ and $P \in \mathrm{PGL}_{n}$. The subset consisting of absolutely irreducible representations is Zariski open in the representation variety, and it is called the "absolutely irreducible representation part" or the "a.i.r. part". The a.i.r. part coincides with the subset consisting of properly stable points of the representation variety by $\mathrm{PGL}_{n}$ in the sense of GIT [2]. We can construct the character variety by taking a universal geometric quotient by $\mathrm{PGL}_{n}$ of the a.i.r. part.

Since the representation variety and the character variety are constructed over $\mathbf{Z}$, they are useful for extending the representation theory over fields or local rings to the one over any commutative rings. Roughly speaking, they connect the "local theory" with the "global theory" on representations. For example, we can apply the character variety to the deformation theory of representations. This is one of the reasons why we work over $\mathbb{Z}$.

The other reason why we work over $\mathbb{Z}$ is that there exist several demands for describing various objects over any commutative ring by using the character varieties. In [9], K. Saito tried to construct a general framework for describing the Teichmüller space of Riemann surfaces as the Fricke space. Since such an object is described as a real algebraic space, so we can not use the usual technique of algebraic geometry over algebraically closed fields. Hence he attempted to construct the character variety over the real number field $\mathbb{R}$ or moreover the integer ring $\mathbb{Z}$. Indeed he constructed the character variety of degree 2 over $\mathbb{Z}$. In this paper we shall construct the character variety over $\mathbb{Z}$ of every degree.

We note that if $\Gamma$ is a finitely generated group, then the character variety for $\Gamma$ is of finite type over $\mathbb{Z}$. If the group $\Gamma$ is a topological group, for example, a Lie group, then the character variety for $\Gamma$ is very huge because it contains non-continuous absolutely irreducible representations. If $\Gamma$ is a Lie 
group, then we had better consider the character variety for the Lie algebra of $\Gamma$ instead of the one for $\Gamma$. Attention is necessary when we consider the character variety for a huge group.

Here we refer to related works of others. One is Donkin's important result in [3]. Donkin showed that the PGL-invariant subring of the coordinate ring of the space of matrices is generated by coefficients of characteristic polynomials of matrices over $\mathbf{Z}$ (Theorem 2.12). This theorem is essential for constructing the character varieties of free groups. Indeed, the PGL-invariant subring is exactly the coordinate ring of the character variety in the free group case, and this result gives rich information about the coordinate ring.

Next we should refer to King's paper [5]. King constructed the moduli spaces of $\theta$-stable or $\theta$-semistable representations of quivers, which have many applications to the moduli of vector bundles and so on. He introduced the notion of $\theta$-stability of modules over the path algebras of quivers. Although the character varieties can be constructed for an arbitrary associative algebra, they are not direct generalization of King's moduli spaces, since the character varieties deal with only absolutely irreducible representations at present. However the character varieties are closely related to his moduli spaces. In this article we construct the character variety independently of King's paper.

As an application of the character variety, we can describe several moduli schemes of vector bundles. Here let us explain a certain relation between the character variety for the free monoid of rank 2 and the moduli of vector bundles over $\mathbf{P}^{2}$. Fix some line $\ell_{0} \subseteq \mathbf{P}^{2}$. Let $\Upsilon_{2}$ be the free monoid with a system of free generators $\{\alpha, \beta\}$. We denote by $\mathbf{M}_{\mathbf{P}^{2}}(r ; 0, n)_{\ell_{0}}^{\mu \text {-stable }}$ the coarse moduli scheme of $\mu$-stable vector bundles over $\mathbf{P}^{2}$ with $c_{1}=0, c_{2}=n, \mathrm{rk}=r$ whose restrictions on $\ell_{0}$ are trivial. We denote by $\mathrm{Ch}_{n}\left(\Upsilon_{2}\right)_{\text {a.i.r. }}^{(r)}$ the coarse moduli scheme of absolutely irreducible representations of degree $n$ for $\Upsilon_{2}$ such that $\operatorname{rk}[\rho(\alpha), \rho(\beta)]=r$, where $[\rho(\alpha), \rho(\beta)]=\rho(\alpha) \rho(\beta)-\rho(\beta) \rho(\alpha)$. Then by the monad construction of vector bundles we see that $\mathbf{M}_{\mathbf{P}^{2}}(r ; 0, n)_{\ell_{0}}^{\mu \text {-stable }}$ is isomorphic to $\mathrm{Ch}_{n}\left(\Upsilon_{2}\right)_{\text {a.i.r }}^{(r)}$ if $n \geq r \geq 2$. It is more natural to consider representations of quivers for studying $\mu$-stable vector bundles with $c_{1}=0$ over $\mathbf{P}^{2}$, but we can not explain the details here. More details can be found in [7].

The basic conventions of this paper are these: Each commutative ring has the identity element 1 . All homomorphisms of commutative rings take 1 to 1 . If $R$ is a commutative ring and if $\wp$ is a prime ideal of $R$, then we denote by $k(\wp)$ the residue field of $\wp$, that is, $R_{\wp} / \wp R_{\wp}$. For a commutative ring $R$, the general linear group is defined as $\mathrm{GL}_{n}(R):=\left\{A \in \mathrm{M}_{n}(R) \mid \operatorname{det}(A) \in R^{\times}\right\}$. The identity matrix of $\mathrm{GL}_{n}(R)$ is denoted by $I_{n}$. For a group $\Gamma$, we denote by $e$ the unit of $\Gamma$. For a scheme $Z$, we denote by $h_{Z}$ the functor $\operatorname{Hom}(\cdot, Z)$ from the category of schemes to the category of sets. We also denote by $\Gamma\left(Z, \mathcal{O}_{Z}\right)$ the ring of global sections on $Z$. 
The author should like to express his gratitude to Professor Masaki Maruyama for instructing him in algebraic geometry, especially in Geometric Invariant Theory and in the theory of vector bundles. The author expresses his thanks to Professor Kyoji Saito for helpful discussions on the paper [9], which motivated the author to construct the character varieties of higher degree; Professor Saito and his paper [9] influenced the author's course in mathematics. The author is grateful to Doctor Akira Ishii for his helpful suggestions on this paper, especially on simplifications of the proofs of several propositions. The author was given rich information on deformation spaces of representations and related topics to the present paper by communication with Professor Yasutaka Ihara. He also pointed out bad manners and errors of the manuscript. The author is sincerely grateful to Professor Ihara. Finally the author expresses his gratitude to the referees for their suggestions on improvements of Theorems 6.12 and 6.13 and for introducing him to several papers [12], [1], and [10].

\section{§1. Main Theorem}

In this section we state the main theorem of this paper: for any group and for any degree, there exists a coarse moduli scheme of equivalence classes of absolutely irreducible representations (Theorem 1.3). We call this moduli scheme the "character variety". To state the main theorem more precisely, we need to prepare some terminology.

If $\phi: R \rightarrow S$ is a homomorphism of commutative rings, then $\phi$ induces the group homomorphism $\phi_{*}: \mathrm{GL}_{n}(R) \rightarrow \mathrm{GL}_{n}(S)$ by sending $\left(a_{i j}\right)$ to $\left(\phi\left(a_{i j}\right)\right)$. In particular, for a commutative ring $R$ and a prime ideal $\wp$ of $R$, the canonical ring homomorphism from $R$ to $k(\wp)$ induces the group homomorphism $\mathrm{GL}_{n}(R) \rightarrow \mathrm{GL}_{n}(k(\wp))$, where $k(\wp)$ is the residue field of $\wp$, that is, $k(\wp)=R_{\wp} / \wp R_{\wp}$.

Definition 1.1. Let $\Gamma$ be a group and $R$ a commutative ring. A map $\rho: \Gamma \rightarrow \mathrm{GL}_{n}(R)$ is called a representation if $\rho$ is a group homomorphism. We say that a representation $\rho: \Gamma \rightarrow \mathrm{GL}_{n}(R)$ is absolutely irreducible if the representation $\rho_{\wp}: \Gamma \rightarrow \mathrm{GL}_{n}(k(\wp))$ is absolutely irreducible for each prime ideal $\wp \in \operatorname{Spec} R$, where $\rho_{\wp}$ is the composite of $\rho$ and the group homomorphism $\mathrm{GL}_{n}(R) \rightarrow \mathrm{GL}_{n}(k(\wp))$. Recall that a representation $\rho: \Gamma \rightarrow \mathrm{GL}_{n}(k)$ with a field $k$ is said to be absolutely irreducible if the representation $\bar{\rho}: \Gamma \rightarrow \mathrm{GL}_{n}(\bar{k})$ induced by $\rho$ is irreducible, where $\bar{k}$ is an algebraic closure of $k$. We abbreviate "absolutely irreducible representation" to "a.i.r.".

By a group homomorphism $\rho: \Gamma \rightarrow \mathrm{GL}_{n}\left(\Gamma\left(X, \mathcal{O}_{X}\right)\right)$, we understand a representation in a scheme $X$. We say that a representation $\rho$ in a scheme $X$ is absolutely irreducible if for each $x \in X$ the representation $\rho_{x}: \Gamma \rightarrow \mathrm{GL}_{n}(k(x))$ is absolutely irreducible. 
Definition 1.2. For two representations $\rho, \rho^{\prime}: \Gamma \rightarrow \mathrm{GL}_{n}(R)$, we say that $\rho$ and $\rho^{\prime}$ are equivalent (or $\rho \sim \rho^{\prime}$ ) if there exists an $R$-algebra isomorphism $\sigma: \mathbf{M}_{n}(R) \rightarrow \mathbf{M}_{n}(R)$ such that $\sigma(\rho(\gamma))=\rho^{\prime}(\gamma)$ for each $\gamma \in \Gamma$.

If $\rho \sim \rho^{\prime}$ and if $\rho$ is absolutely irreducible, then so is $\rho^{\prime}$. If $R$ is a field, then $\rho \sim \rho^{\prime}$ if and only if $\rho=P \rho^{\prime} P^{-1}$ for some $P \in \mathrm{GL}_{n}(R)$ because of the Skolem-Noether Theorem.

For two representations $\rho$ and $\rho^{\prime}$ in a scheme $X$, we say that $\rho$ and $\rho^{\prime}$ are equivalent if there exists an $\mathcal{O}_{X}$-algebra isomorphism $\sigma: \mathbf{M}_{n}\left(\mathcal{O}_{X}\right) \rightarrow \mathbf{M}_{n}\left(\mathcal{O}_{X}\right)$ such that $\sigma(\rho(\gamma))=\rho^{\prime}(\gamma)$ for each $\gamma \in \Gamma$.

Now we introduce the moduli functor related to the representation theory. We define the contravariant functor $\mathscr{E}_{q} \mathscr{A} \mathscr{I} \mathscr{R}_{n}(\Gamma)$ from the category of schemes to that of sets:

$$
\begin{aligned}
\mathscr{E}_{q} \mathscr{A} \mathscr{I} \mathscr{R}_{n}(\Gamma):(\text { Sch }) & \rightarrow(\text { Sets }) \\
X & \mapsto\left\{\rho: \Gamma \rightarrow \mathrm{GL}_{n}\left(\Gamma\left(X, \mathcal{O}_{X}\right)\right) \text { an a.i.r. }\right\} / \sim .
\end{aligned}
$$

With the functor above we can now state the main theorem:

Theorem 1.3 (The Main Theorem). There exists a coarse moduli scheme $\mathrm{Ch}_{n}(\Gamma)_{\text {a.i.r. }}$ over $\mathbf{Z}$ associated to the moduli functor $\mathscr{E}_{q} \mathscr{A} \mathscr{I} \mathscr{R}_{n}(\Gamma)$. In other words, there exist a separated scheme $\mathrm{Ch}_{n}(\Gamma)_{\text {a.i.r. }}$ over $\mathbf{Z}$ and a natural transformation $\tau: \mathscr{E}_{q} \mathscr{A} \mathscr{I} \mathscr{R}_{n}(\Gamma) \rightarrow h_{\mathrm{Ch}_{n}(\Gamma)_{\text {a I }}}$ satisfying the following two conditions.

(i) For any scheme $Z$, the natural transformation $\tau$ induces the following isomorphism:

$$
\tau: \operatorname{Hom}\left(\mathscr{E}_{q} \mathscr{A} \mathscr{I} \mathscr{R}_{n}(\Gamma), h_{Z}\right) \cong \operatorname{Hom}\left(h_{\mathrm{Ch}_{n}(\Gamma)_{\mathrm{a} 1 \mathrm{r}}}, h_{Z}\right) .
$$

(ii) For any algebraically closed field $\Omega$, the morphism

$$
\tau: \mathscr{E}_{q} \mathscr{A} \mathscr{I} \mathscr{R}_{n}(\Gamma)(\operatorname{Spec} \Omega) \rightarrow h_{\mathrm{Ch}_{n}(\Gamma)_{\mathrm{a} 1 \mathrm{r}}}(\operatorname{Spec} \Omega)
$$

is bijective.

We shall call the scheme $\mathrm{Ch}_{n}(\Gamma)_{\text {a.i.r. }}$ the (absolutely irreducible) character variety of degree $n$ for $\Gamma$.

Here we give an outline of the proof of the main theorem. We introduce the representation variety $\operatorname{Rep}_{n}(\Gamma)$ for each group $\Gamma$ in $\S 2$. The representation variety $\operatorname{Rep}_{n}(\Gamma)$ is the affine scheme parameterizing the representations of degree $n$ for $\Gamma$. The group scheme $\mathrm{PGL}_{n}$ canonically acts on the representation variety by $\rho \mapsto P^{-1} \rho P$ for $\rho \in \operatorname{Rep}_{n}(\Gamma)$ and $P \in \mathrm{PGL}_{n}$. The coordinate ring $\mathrm{A}_{n}(\Gamma)$ of $\operatorname{Rep}_{n}(\Gamma)$ has two subring $\mathrm{A}_{n}(\Gamma)^{\mathrm{Ch}} \subseteq \mathrm{A}_{n}(\Gamma)^{\mathrm{PGL}_{n}}$, where the first one is generated by all coefficients of the characteristic polynomials of the universal representation, and the second one is obtained by taking invariants under the action of $\mathrm{PGL}_{n}$. The respective spectra of $\mathrm{A}_{n}(\Gamma)^{\mathrm{Ch}}$ and $\mathrm{A}_{n}(\Gamma)^{\mathrm{PGL}_{n}}$ are denoted 
by $\mathrm{Ch}_{n}^{0}(\Gamma)$ and $\mathrm{Ch}_{n}(\Gamma)$. In $\S 3$ we shall show that the subset consisting of absolutely irreducible representations (the a.i.r. part, which is denoted by $\left.\operatorname{Rep}_{n}(\Gamma)_{\text {a.i.r. }}\right)$ is Zariski open in the representation variety. For describing the a.i.r. part we introduce a $\mathrm{PGL}_{n}$-invariant $\Delta$ called a discriminant. The a.i.r. parts of $\mathrm{Ch}_{n}^{0}(\Gamma)$ and $\mathrm{Ch}_{n}(\Gamma)$ are similarly defined, and they are denoted by $\mathrm{Ch}_{n}^{0}(\Gamma)_{\text {a.i.r. }}$ and $\mathrm{Ch}_{n}(\Gamma)_{\text {a.i.r. }}$, respectively. Then there exists a natural morphism $\pi_{n, \Gamma \text {, air }}: \operatorname{Rep}_{n}(\Gamma)_{\text {a.i.r. }} \rightarrow \mathrm{Ch}_{n}^{0}(\Gamma)_{\text {a.i.r. }}$. We construct the character variety by taking a universal geometric quotient of $\operatorname{Rep}_{n}(\Gamma)_{\text {a.i.r. }}$ by $\mathrm{PGL}_{n}$. The latter part of this paper $(\S 4-\S 6)$ is devoted to proving that $\pi_{n, \Gamma \text {, air }}$ is a universal geometric quotient. For proving this claim, we only have to prove the case that $\Gamma$ is a free group by Theorem 5.1: any morphism $\pi_{n, \Gamma, \Delta}: \operatorname{Rep}_{n}(\Gamma)_{\Delta} \rightarrow \mathrm{Ch}_{n}^{0}(\Gamma)_{\Delta}$ is obtained by the base change of the special morphism $\pi_{n, F_{n}, \tilde{\Delta}}: \operatorname{Rep}_{n}\left(\mathrm{~F}_{n^{2}}\right)_{\tilde{\Delta}} \rightarrow \mathrm{Ch}_{n}^{0}\left(\mathrm{~F}_{n^{2}}\right)_{\tilde{\Delta}}$. In $\S 6$ we shall prove that the action of $\mathrm{PGL}_{n}$ on the a.i.r. part is free by using the Skolem-Noether theorem and Theorem 4.1. The explicit formula in Theorem 4.1 implies that any matrix can be reconstructed by its $\mathrm{PGL}_{n}$-invariants. From the freeness of the action, we see that the morphism $\pi_{n, \Gamma \text {, air }}$ is a universal geometric quotient (we note that $\pi_{n, \Gamma \text {,air }}$ is faithfully flat). Therefore we see that $\operatorname{Ch}_{n}^{0}(\Gamma)_{\text {a.i.r. }}$ is the character variety. We remark that $\pi_{n, \Gamma \text {,arr }}$ is a $\mathrm{PGL}_{n}$-principal fiber bundle and that $\mathrm{Ch}_{n}^{0}(\Gamma)_{\text {a.i.r. }}$ coincides with $\mathrm{Ch}_{n}(\Gamma)_{\text {a.i.r. }}$.

Remark 1.4. Let $\Upsilon$ be a semigroup or a monoid. A map $\rho: \Upsilon \rightarrow \mathbf{M}_{n}(R)$ is called a representation of $\Upsilon$ if $\rho$ is a semigroup (or resp. a monoid) homomorphism. The contravariant functor $\mathscr{E}_{q} \mathscr{A} \mathscr{I} \mathscr{R}_{n}(\Upsilon)$ for $\Upsilon$ is defined in the similar way as the group case. Then we can also show that a coarse moduli scheme $\mathrm{Ch}_{n}(\Upsilon)_{\text {a.i.r. }}$ exists for the moduli functor $\mathscr{E}_{q} \mathscr{A} \mathscr{I} \mathscr{R}_{n}(\Upsilon)$.

Remark 1.5. Let $A$ be an associative algebra over a commutative ring $R$. For a commutative $R$-algebra $S$, we say that a map $\rho: A \rightarrow \mathrm{M}_{n}(S)$ is a representation of $A$ in $\mathrm{M}_{n}(S)$ if $\rho$ is an $R$-algebra homomorphism. Two representations $\rho, \rho^{\prime}: A \rightarrow \mathbf{M}_{n}(S)$ are called equivalent (or $\rho \sim \rho^{\prime}$ ) if there exists an $S$-algebra isomorphism $\sigma: \mathrm{M}_{n}(S) \rightarrow \mathrm{M}_{n}(S)$ such that $\sigma(\rho(a))=\rho^{\prime}(a)$ for each $a \in A$. We say that a representation $\rho: A \rightarrow \mathrm{M}_{n}(S)$ is absolutely irreducible if the representation $\rho_{\wp}: A \rightarrow \mathrm{M}_{n}(k(\wp))$ induced by $\rho$ is absolutely irreducible for each prime ideal $\wp \in \operatorname{Spec}(S)$. We introduce the moduli functor $\mathscr{E}_{q} \mathscr{A} \mathscr{I} \mathscr{R}_{n}(A)$ from the category of schemes over $R$ to that of sets in the similar way as the group case.

$$
\begin{aligned}
\mathscr{E}_{q} \mathscr{A} \mathscr{I} \mathscr{R}_{n}(A):(\mathbf{S c h} / R) & \rightarrow(\text { Sets }) \\
X & \mapsto\left\{\rho: A \rightarrow \mathbf{M}_{n}\left(\mathcal{O}_{X}(X)\right) \text { an a.i.r. }\right\} / \sim
\end{aligned}
$$

We can construct the coarse moduli scheme $\mathrm{Ch}_{n}(A)_{\text {a.i.r. }}$ associated to $\mathscr{E}_{q} \mathscr{A} \mathscr{I} \mathscr{R}_{n}(A)$ as a scheme over $R$ in the similar way as Theorem 1.3. 
For a Lie algebra $g$ over a commutative ring $R$, we can also construct the coarse moduli scheme of equivalence classes of absolutely irreducible representations of $\mathfrak{g}$. Indeed, it can be constructed as the character variety for the universal enveloping algebra of $\mathfrak{g}$.

\section{§2. Representation Varieties}

To construct the character variety, we need to introduce the representation variety. The representation variety of degree $n$ for a group $\Gamma$ is the affine scheme parameterizing the representations of degree $n$ for $\Gamma$. In this section we give basic results on the representation variety and introduce several schemes, which turn out to be the character varieties.

Definition 2.1 (cf. [9]). Let $\Gamma$ be a group and $n$ a positive integer. Let us consider the polynomial ring $\mathbb{Z}\left[a_{i j}(\gamma) \mid \gamma \in \Gamma, 1 \leq i, j \leq n\right]$, where the symbols $a_{i j}(\gamma)$ are independent variables. By $I(\Gamma)$, we understand the ideal of the polynomial ring defined by

$$
I(\Gamma):=\left(a_{i j}(e)-\delta_{i j}, a_{i j}(\gamma \delta)-\sum_{k=1}^{n} a_{i k}(\gamma) a_{k j}(\delta) \mid \gamma, \delta \in \Gamma, 1 \leq i, j \leq n\right) .
$$

Then we define the representation ring $\mathrm{A}_{n}(\Gamma)$ of degree $n$ for $\Gamma$ by

$$
\mathrm{A}_{n}(\Gamma):=\mathbf{Z}\left[a_{i j}(\gamma) \mid \gamma \in \Gamma, 1 \leq i, j \leq n\right] / I(\Gamma) .
$$

We call the spectrum of $\mathrm{A}_{n}(\Gamma)$ the representation variety of degree $n$ for $\Gamma$, denoted by $\operatorname{Rep}_{n}(\Gamma)$.

Definition 2.2. With the same notation as Definition 2.1, we define the universal representation $\sigma_{\Gamma}$ of degree $n$ for $\Gamma$ by

$$
\begin{aligned}
\sigma_{\Gamma}: \Gamma & \rightarrow \mathrm{GL}_{n}\left(\mathrm{~A}_{n}(\Gamma)\right) \\
\gamma & \mapsto\left(a_{i j}(\gamma)\right)_{1 \leq i, j \leq n} .
\end{aligned}
$$

Here we regard $a_{i j}(\gamma)$ as an element of the ring $\mathrm{A}_{n}(\Gamma)$ through the canonical projection $\mathbf{Z}\left[a_{i j}(\gamma) \mid \gamma \in \Gamma, 1 \leq i, j \leq n\right] \rightarrow \mathrm{A}_{n}(\Gamma)$. We see that the map $\sigma_{\Gamma}$ is a representation because of the definition of $\mathrm{A}_{n}(\Gamma)$.

The next proposition tells us the reason why the scheme $\operatorname{Rep}_{n}(\Gamma)$ and the representation $\sigma_{\Gamma}$ are called the representation variety and the universal representation, respectively.

Proposition 2.3. The affine scheme $\operatorname{Rep}_{n}(\Gamma)$ represents the following contravariant functor from the category of schemes to that of sets: 


$$
\begin{aligned}
\operatorname{Rep}_{n}(\Gamma):(\text { Sch }) & \rightarrow(\text { Sets }) \\
X & \mapsto\left\{\rho: \Gamma \rightarrow \mathrm{GL}_{n}\left(\Gamma\left(X, \mathcal{O}_{X}\right)\right) \mid \rho \text { is a representation }\right\},
\end{aligned}
$$

where $\Gamma\left(X, \mathcal{O}_{X}\right)$ is the ring of global sections on $X$.

Proof. For a scheme $X$ we consider the following map:

$$
\begin{aligned}
\operatorname{Hom}_{\text {ring }}\left(\mathrm{A}_{n}(\Gamma), \Gamma\left(X, \mathcal{O}_{X}\right)\right) & \rightarrow \operatorname{Hom}_{\text {group }}\left(\Gamma, \mathrm{GL}_{n}\left(\Gamma\left(X, \mathcal{O}_{X}\right)\right)\right) \\
\phi & \mapsto \phi_{*} \circ \sigma_{\Gamma},
\end{aligned}
$$

where $\phi_{*}: \mathrm{GL}_{n}\left(\mathrm{~A}_{n}(\Gamma)\right) \rightarrow \mathrm{GL}_{n}\left(\Gamma\left(X, \mathcal{O}_{X}\right)\right)$ is the group homomorphism induced by $\phi$. We easily see that the map is an isomorphism by the definition of $\sigma_{\Gamma}$. From the isomorphism

$$
\operatorname{Hom}\left(X, \operatorname{Rep}_{n}(\Gamma)\right) \cong \operatorname{Hom}_{\text {ring }}\left(\mathrm{A}_{n}(\Gamma), \Gamma\left(X, \mathcal{O}_{X}\right)\right),
$$

we obtain the statement.

Remark 2.4. In the semigroup (or monoid) case we can also define the representation variety with the universal property as in Proposition 2.3.

The name of "representation variety" is due to K. Saito in [9]. Remark that representation varieties are neither irreducible nor reduced in general. However we will use this name and the name of "character variety" according to [9].

Remark 2.5. If $\phi: \Gamma_{1} \rightarrow \Gamma_{2}$ is a group homomorphism, then $\phi$ induces the ring homomorphism

$$
\begin{aligned}
\phi_{*}: \mathrm{A}_{n}\left(\Gamma_{1}\right) & \rightarrow \mathrm{A}_{n}\left(\Gamma_{2}\right) \\
a_{i j}(\gamma) & \mapsto a_{i j}(\phi(\gamma)) .
\end{aligned}
$$

Hence the group homomorphism $\phi$ induces the morphism of schemes $\phi^{*}: \operatorname{Rep}_{n}\left(\Gamma_{2}\right) \rightarrow \operatorname{Rep}_{n}\left(\Gamma_{1}\right)$. If $\phi$ is surjective, then $\phi_{*}$ is also surjective; in particular $\phi^{*}$ is a closed immersion.

We now introduce some examples of representation varieties.

Example 2.6 (cf. [8]). Let $\mathbb{Z}\left[\mathrm{M}_{n}^{\oplus m}\right]$ be the coordinate ring for the space of $m$-tuples of $n \times n$ matrices $\left(A_{1}, A_{2}, \ldots, A_{m}\right)$, that is,

$$
\mathbb{Z}\left[\mathbf{M}_{n}^{\oplus m}\right]:=\mathbb{Z}\left[a_{i j}^{k} \mid 1 \leq i, j \leq n, 1 \leq k \leq m\right],
$$

where $A_{k}=\left(a_{i j}^{k}\right)_{1 \leq i, j \leq n}$. The ring $\mathbb{Z}\left[\mathbf{M}_{n}^{\oplus m}\right]$ is isomorphic to the polynomial ring in $n^{2} m$ variables over $\mathbb{Z}$. Set $\operatorname{det} A:=\prod_{k=1}^{n} \operatorname{det} A_{k}$. Let $\mathbb{Z}\left[\mathbf{M}_{n}^{\oplus m}\right]_{\operatorname{det} A}$ denote the localization of $\mathbb{Z}\left[\mathbf{M}_{n}^{\oplus m}\right]$ by $\operatorname{det} A$.

For the free group $\mathrm{F}_{m}$ of rank $m$ with a system of free generators $\left\{\alpha_{1}, \ldots, \alpha_{m}\right\}$, we consider the following ring homomorphism: 


$$
\begin{aligned}
\mathbf{Z}\left[\mathrm{M}_{n}^{\oplus m}\right]_{\operatorname{det} A} & \rightarrow \mathrm{A}_{n}\left(\mathrm{~F}_{m}\right) \\
a_{i j}^{k} & \mapsto a_{i j}\left(\alpha_{k}\right) .
\end{aligned}
$$

It is easy to see that the homomorphism is an isomorphism, hence the spectrum of $\mathbb{Z}\left[\mathbf{M}_{n}^{\oplus m}\right]_{\operatorname{det} A}$ is isomorphic to $\operatorname{Rep}_{n}\left(\mathrm{~F}_{m}\right)$. We can also see that $\operatorname{Spec} \mathbf{Z}\left[\mathbf{M}_{n}^{\oplus m}\right]$ is isomorphic to $\operatorname{Rep}_{n}\left(\Upsilon_{m}\right)$, where $\Upsilon_{m}$ is the free monoid of rank $m$.

Example 2.7. Let us consider the case that $\Gamma$ is an arbitrary group. Suppose that $\Gamma$ is expressed as $\mathrm{F}_{I} / R$, where $\mathrm{F}_{I}$ is a free group with a system of free generators $I$ and $R$ is a set of fundamental relations. Let $\mathbf{Z}\left[\prod_{I} \mathbf{M}_{n}\right]$ denote the coordinate ring for the space of \#I-tuples of $n \times n$ matrices $\left(A_{i}\right)_{i \in I}$. Let $\operatorname{det} A$ denote the multiplicative system in $\mathbf{Z}\left[\prod_{I} \mathbf{M}_{n}\right]$ generated by $\left\{\operatorname{det} A_{i}\right\}_{i \in I}$. As in Example 2.6 we see that $\mathbf{Z}\left[\prod_{I} \mathbf{M}_{n}\right]_{\operatorname{det} A} \cong \mathrm{A}_{n}\left(\mathrm{~F}_{I}\right)$, hence $\operatorname{Spec}\left(\mathbb{Z}\left[\Pi_{I} \mathrm{M}_{n}\right]_{\operatorname{det} A}\right)$ is isomorphic to $\operatorname{Rep}_{n}\left(\mathrm{~F}_{I}\right)$. Let $\sigma_{\mathrm{F}_{I}}: \mathrm{F}_{I} \rightarrow$ $\mathrm{GL}_{n}\left(\mathbf{Z}\left[\Pi_{I} \mathbf{M}_{n}\right]_{\operatorname{det} A}\right)$ denote the universal representation of degree $n$ for $\mathrm{F}_{I}$. Then the representation ring of degree $n$ for $\Gamma$ is isomorphic to $\mathbf{Z}\left[\prod_{I} \mathbf{M}_{n}\right]_{\operatorname{det} A} / J$, where $J$ is the ideal of $\mathbf{Z}\left[\prod_{I} \mathbf{M}_{n}\right]_{\operatorname{det} A}$ generated by all entries of matrices $\left\{\sigma_{\mathrm{F}_{I}}(\delta)-I_{n} \mid \delta \in R\right\}$. Hence we have $\operatorname{Rep}_{n}(\Gamma) \cong \operatorname{Spec} \mathbb{Z}\left[\prod_{I} \mathbf{M}_{n}\right]_{\operatorname{det} A} / J$.

Remark 2.8. We can easily see that $\operatorname{Rep}_{n}(\Gamma)$ is of finite type over $\mathbf{Z}$ if $\Gamma$ is a finitely generated group, and that in general case $\operatorname{Rep}_{n}(\Gamma)$ is the projective limit of the $\operatorname{Rep}_{n}\left(\Gamma^{\prime}\right)$, where $\Gamma^{\prime}$ runs through the finitely generated subgroups of $\Gamma$.

We recall the group scheme $\left.\mathrm{PGL}_{n}:=\operatorname{Spec} Z x_{i j} \mid 1 \leq i, j \leq n\right]_{\left(\operatorname{det}\left(x_{i}\right)\right)}$ over $\mathbf{Z}$. Here we denote by $\left(\mathrm{A}\left(\mathrm{PGL}_{n}\right):=\right) \mathbb{Z}\left[x_{i j} \mid 1 \leq i, j \leq n\right]_{\left(\operatorname{det}\left(x_{l}\right)\right)}$ the subring of elements of degree 0 in the graded ring $\mathbf{Z}\left[x_{i j} \mid 1 \leq i, j \leq n\right]_{\operatorname{det}\left(x_{i}\right)}$. There exists a natural action of $\mathrm{PGL}_{n}$ on the representation variety $\operatorname{Rep}_{n}(\Gamma)$ :

$$
\begin{aligned}
\operatorname{Ad}: \operatorname{Rep}_{n}(\Gamma) \times \operatorname{PGL}_{n} & \rightarrow \operatorname{Rep}_{n}(\Gamma) \\
(\rho, P) & \mapsto P^{-1} \rho P .
\end{aligned}
$$

We denote by $\mathrm{Ad}^{*}: \mathrm{A}_{n}(\Gamma) \rightarrow \mathrm{A}_{n}(\Gamma) \otimes_{\mathrm{Z}} \mathrm{A}\left(\mathrm{PGL}_{n}\right)$ the ring homomorphism associated to Ad.

We introduce some notation about $\mathrm{PGL}_{n}$-invariants.

Notation 2.9. Let $R$ be a commutative ring. For $A \in \mathrm{M}_{n}(R)$, we define $c_{1}(A), c_{2}(A), \ldots, c_{n}(A) \in R$ by

$$
\operatorname{det}\left(x I_{n}-A\right)=x^{n}-c_{1}(A) x^{n-1}+c_{2}(A) x^{n-2}-\cdots+(-1)^{n} c_{n}(A) .
$$

For the universal representation $\sigma_{\Gamma}: \Gamma \rightarrow \mathrm{GL}_{n}\left(\mathrm{~A}_{n}(\Gamma)\right)$, we simply denote $c_{i}\left(\sigma_{\Gamma}(\gamma)\right)$ by $c_{i}(\gamma)$. We specially denote $c_{1}(\gamma)$ and $c_{n}(\gamma)$ by $s(\gamma)$ and $d(\gamma)$, respectively. The letters $s$ and $d$ mean the initial of "Spur" and "Determinante", respectively. 
Definition 2.10. The subalgebra $\mathrm{A}_{n}(\Gamma)^{\mathrm{Ch}}$ of the representation ring $\mathrm{A}_{n}(\Gamma)$ is defined as the subalgebra generated by $\left\{c_{i}(\gamma) \mid 1 \leq i \leq n, \gamma \in \Gamma\right\}$ over $\mathbb{Z}$. The subalgebra of $\mathrm{PGL}_{n}$-invariants $\mathrm{A}_{n}(\Gamma)^{\mathrm{PGL}_{n}}$ of $\mathrm{A}_{n}(\Gamma)$ is defined by

$$
\mathrm{A}_{n}(\Gamma)^{\mathrm{PGL}_{n}}:=\left\{x \in \mathrm{A}_{n}(\Gamma) \mid \mathrm{Ad}^{*}(x)=x \otimes 1 \text { in } \mathrm{A}_{n}(\Gamma) \otimes_{\mathbf{Z}} \mathrm{A}\left(\mathrm{PGL}_{n}\right)\right\} .
$$

For a commutative ring $R$, we also define $\left(\mathrm{A}_{n}(\Gamma) \otimes_{\mathrm{Z}} R\right)^{\mathrm{PGL}_{n}}$ by

$$
\left(\mathrm{A}_{n}(\Gamma) \otimes_{\mathbf{Z}} R\right)^{\mathrm{PGL}_{n}}:=\left\{x \in \mathrm{A}_{n}(\Gamma) \otimes_{\mathbf{Z}} R \mid \operatorname{Ad}_{R}^{*}(x)=x \otimes 1\right\},
$$

where $\operatorname{Ad}_{R}^{*}: \mathrm{A}_{n}(\Gamma) \otimes_{\mathrm{Z}} R \rightarrow\left(\mathrm{A}_{n}(\Gamma) \otimes_{\mathrm{Z}} R\right) \otimes_{\mathrm{Z}} \mathrm{A}\left(\mathrm{PGL}_{n}\right)$ is the morphism induced by Ad. As in the group case, we define two subalgebras $\mathrm{A}_{n}(\Upsilon)^{\mathrm{Ch}}$ and $\mathrm{A}_{n}(\Upsilon)^{\mathrm{PGL}_{n}}$ of the representation ring $\mathrm{A}_{n}(\Upsilon)$ for a semigroup (or a monoid) $\Upsilon$.

Notation 2.11. For a group $\Gamma$, we put

$$
\begin{aligned}
& \mathrm{Ch}_{n}(\Gamma):=\operatorname{Spec}_{n}(\Gamma)^{\mathrm{PGL}_{n}}, \\
& \mathrm{Ch}_{n}^{0}(\Gamma):=\operatorname{Spec}_{n}(\Gamma)^{\mathrm{Ch}} .
\end{aligned}
$$

It is clear that $\mathrm{A}_{n}(\Gamma)^{\mathrm{Ch}} \subseteq \mathrm{A}_{n}(\Gamma)^{\mathrm{PGL}_{n}} \subseteq \mathrm{A}_{n}(\Gamma)$. The inclusions of rings induce the following morphisms, whose composite will be called $\pi_{n, \Gamma}$ :

$$
\pi_{n, \Gamma}: \operatorname{Rep}_{n}(\Gamma) \rightarrow \mathrm{Ch}_{n}(\Gamma) \rightarrow \mathrm{Ch}_{n}^{0}(\Gamma)
$$

By Example 2.6, we see that the representation ring $\mathrm{A}_{n}\left(\Upsilon_{m}\right)$ for the free monoid $\Upsilon_{m}$ is isomorphic to $\mathbf{Z}\left[\mathbf{M}_{n}^{\oplus m}\right]$, that is, the coordinate ring for the space of $m$-tuples of $n \times n$ matrices $\left(A_{1}, \ldots, A_{m}\right)$. We denote $\mathrm{A}_{n}\left(\Upsilon_{m}\right)^{\mathrm{PGL}_{n}}$ by $\mathbb{Z}\left[\mathrm{M}_{n}^{\oplus m}\right]^{\mathrm{PGL}_{n}}$. Similarly we denote $\left(\mathrm{A}_{n}\left(\Upsilon_{m}\right) \otimes_{\mathbf{Z}} R\right)^{\mathrm{PGL}_{n}}$ by $R\left[\mathrm{M}_{n}^{\oplus m}\right]^{\mathrm{PGL}_{n}}$ for a commutative ring $R$. Here we quote an important theorem about the ring $\mathbb{Z}\left[\mathbf{M}_{n}^{\oplus m}\right]^{\mathrm{PGL}_{n}}$ from [3].

Theorem 2.12 (S. Donkin [3]). In the same notation as above, the invariant ring $\mathbb{Z}\left[\mathrm{M}_{n}^{\oplus m}\right]^{\mathrm{PGL}_{n}}$ is generated by $\left\{c_{j}\left(A_{i_{1}} A_{i_{2}} \cdots A_{i_{1}}\right) \mid 1 \leq j \leq n\right.$ and $\left.i_{1}, i_{2}, \ldots, i_{r} \in\{1,2, \ldots, m\}\right\}$ as a $\mathbb{Z}$-algebra. Moreover if $\Omega$ is any algebraically closed field, then $\Omega\left[\mathrm{M}_{n}^{\oplus m}\right]^{\mathrm{PGL}_{n}} \cong \mathbb{Z}\left[\mathrm{M}_{n}^{\oplus m}\right]^{\mathrm{PGL}_{n}} \otimes_{\mathrm{Z}} \Omega$.

The next corollary immediately follows from this theorem.

Corollary 2.13. For the free group $\mathrm{F}_{m}$ of rank $m$, we have $\mathrm{A}_{n}\left(\mathrm{~F}_{m}\right)^{\mathrm{Ch}}=$ $\mathrm{A}_{n}\left(\mathrm{~F}_{m}\right)^{\mathrm{PGL}_{n}}$.

From Corollary 2.13 we conclude that two subalgebras $\mathrm{A}_{n}(\Gamma)^{\mathrm{PGL}_{n}}$ and $\mathrm{A}_{n}(\Gamma)^{\mathrm{Ch}}$ coincide for finitely generated free groups $\Gamma$. In general case we shall later see that they coincide at least on the absolutely irreducible representation part; it is not yet known whether two algebras coincide outside the a.i.r. part for an arbitrary group. 


\section{§3. Discriminants}

In this section, we describe the subset consisting of absolutely irreducible representations in the representation variety. We call this subset the "absolutely irreducible representation part" or the "a.i.r. part". To describe it, we need an invariant $\Delta$ called a discriminant. The discriminant is defined for $n^{2}$ elements of a group when we consider the representation variety of degree $n$. The a.i.r. part is a Zariski open subset where at least one discriminant does not vanish.

Definition 3.1. Let us consider ordered $n^{2}$ matrices $A_{1}, A_{2}, \ldots, A_{n^{2}} \in \mathrm{M}_{n}(R)$ with a commutative ring $R$. Let $A_{k}=\left(a_{i j}^{(k)}\right)_{1 \leq i, j \leq n}$. The discriminant $\Delta\left(A_{1}, A_{2}, \ldots, A_{n^{2}}\right)$ for $A_{1}, A_{2}, \ldots, A_{n^{2}}$ is defined as the determinant of the matrix

$$
\left(\begin{array}{ccccccccccc}
a_{11}^{(1)} & a_{12}^{(1)} & \ldots & a_{1 n}^{(1)} & a_{21}^{(1)} & \ldots & a_{2 n}^{(1)} & \ldots & a_{n 1}^{(1)} & \ldots & a_{n n}^{(1)} \\
a_{11}^{(2)} & a_{12}^{(2)} & \ldots & a_{1 n}^{(2)} & a_{21}^{(2)} & \ldots & a_{2 n}^{(2)} & \ldots & a_{n 1}^{(2)} & \ldots & a_{n n}^{(2)} \\
\vdots & \vdots & \ldots & \vdots & \vdots & \ldots & \vdots & \ldots & \vdots & \ldots & \vdots \\
a_{11}^{\left(n^{2}\right)} & a_{12}^{\left(n^{2}\right)} & \ldots & a_{1 n}^{\left(n^{2}\right)} & a_{21}^{\left(n^{2}\right)} & \ldots & a_{2 n}^{\left(n^{2}\right)} & \ldots & a_{n 1}^{\left(n^{2}\right)} & \ldots & a_{n n}^{\left(n^{2}\right)}
\end{array}\right)
$$

Let $\alpha_{1}, \alpha_{2}, \ldots, \alpha_{n^{2}}$ be $n^{2}$ ordered elements of a group $\Gamma$. The discriminant $\Delta\left(\alpha_{1}, \alpha_{2}, \ldots, \alpha_{n^{2}}\right)$ for $\alpha_{1}, \alpha_{2}, \ldots, \alpha_{n^{2}}$ is defined by

$$
\Delta\left(\alpha_{1}, \alpha_{2}, \ldots, \alpha_{n^{2}}\right):=\Delta\left(\sigma_{\Gamma}\left(\alpha_{1}\right), \sigma_{\Gamma}\left(\alpha_{2}\right), \ldots, \sigma_{\Gamma}\left(\alpha_{n^{2}}\right)\right) \in \mathrm{A}_{n}(\Gamma),
$$

where $\sigma_{\Gamma}$ is the universal representation of degree $n$ for $\Gamma$.

Remark 3.2. For $A_{1}, A_{2}, \ldots, A_{n^{2}} \in \mathrm{M}_{n}(R)$, we see that

$$
\operatorname{det}\left(\operatorname{tr}\left(A_{i} A_{j}\right)\right)_{1 \leq i, j \leq n^{2}}=(-1)^{n(n-1) / 2} \Delta\left(A_{1}, A_{2}, \ldots, A_{n^{2}}\right)^{2} .
$$

Indeed, consider the following equality and take the determinants of the both sides:

$$
\begin{aligned}
& \left(\begin{array}{cccc}
\operatorname{tr}\left(A_{1} A_{1}\right) & \operatorname{tr}\left(A_{1} A_{2}\right) & \ldots & \operatorname{tr}\left(A_{1} A_{n^{2}}\right) \\
\operatorname{tr}\left(A_{2} A_{1}\right) & \operatorname{tr}\left(A_{2} A_{2}\right) & \ldots & \operatorname{tr}\left(A_{2} A_{n^{2}}\right) \\
\vdots & \vdots & \ldots & \vdots \\
\operatorname{tr}\left(A_{n^{2}} A_{1}\right) & \operatorname{tr}\left(A_{n^{2}} A_{2}\right) & \ldots & \operatorname{tr}\left(A_{n^{2}} A_{n^{2}}\right)
\end{array}\right) \\
& =\left(\begin{array}{cccc}
a_{11}^{(1)} & a_{12}^{(1)} & \ldots & a_{n n}^{(1)} \\
a_{11}^{(2)} & a_{12}^{(2)} & \ldots & a_{n n}^{(2)} \\
\vdots & \vdots & \ldots & \vdots \\
a_{11}^{\left(n^{2}\right)} & a_{12}^{\left(n^{2}\right)} & \ldots & a_{n n}^{\left(n^{2}\right)}
\end{array}\right)\left(\begin{array}{cccc}
a_{11}^{(1)} & a_{11}^{(2)} & \ldots & a_{11}^{\left(n^{2}\right)} \\
a_{21}^{(1)} & a_{21}^{(2)} & \ldots & a_{21}^{\left(n^{2}\right)} \\
\vdots & \vdots & \ldots & \vdots \\
a_{n n}^{(1)} & a_{n n}^{(2)} & \ldots & a_{n n}^{\left(n^{2}\right)}
\end{array}\right) .
\end{aligned}
$$

Then we obtain the formula. 
By the discriminant we understand not $\operatorname{det}\left(\operatorname{tr}\left(A_{i} A_{j}\right)\right)_{1 \leq i, j \leq n^{2}}$ but $\Delta\left(A_{1}, A_{2}, \ldots, A_{n^{2}}\right)$ in this paper. For investigating the complement of the absolutely irreducible representation part in the future (we do not go into the detail in this paper), the polynomial $\Delta\left(A_{1}, A_{2}, \ldots, A_{n^{2}}\right)$ is more reasonable than $\operatorname{det}\left(\operatorname{tr}\left(A_{i} A_{j}\right)\right)_{1 \leq i, j \leq n^{2}}$. Indeed the polynomial $\operatorname{det}\left(\operatorname{tr}\left(A_{i} A_{j}\right)\right)$ is always reducible, and hence it is not suitable for describing the complement of the a.i.r. part.

For $A_{i} \in \mathrm{M}_{n}(R)$, the discriminant $\Delta\left(A_{1}, A_{2}, \ldots, A_{n^{2}}\right)$ is invertible in $R$ if and only if $\left\{A_{1}, A_{2}, \ldots, A_{n^{2}}\right\}$ is a free basis of $\mathrm{M}_{n}(R)$ over $R$. The definition of the discriminant $\Delta\left(A_{1}, \ldots, A_{n^{2}}\right)$ depends on the order of $A_{1}, \ldots, A_{n^{2}}$. Indeed, if $\sigma$ is an element of the symmetric group $\mathfrak{\Xi}_{n^{2}}$, then $\Delta\left(A_{\sigma(1)}, A_{\sigma(2)}, \ldots, A_{\sigma\left(n^{2}\right)}\right)=$ $\operatorname{sgn}(\sigma) \Delta\left(A_{1}, A_{2}, \ldots, A_{n^{2}}\right)$. However whether the discriminant $\Delta$ is invertible depends only on the set $\left\{A_{1}, A_{2}, \ldots, A_{n^{2}}\right\}$.

We see that the discriminant $\Delta$ is $\mathrm{PGL}_{n}$-invariant from the next proposition.

Proposition 3.3. The discriminant $\Delta\left(\alpha_{1}, \ldots, \alpha_{n^{2}}\right)$ is an element of $\mathrm{A}_{n}(\Gamma)^{\mathrm{Ch}}$ for $\alpha_{1}, \ldots, \alpha_{n^{2}} \in \Gamma$.

Proof. Let $\mathrm{F}_{n^{2}}$ be the free group of rank $n^{2}$ with a system of free generators $\left\{\tilde{\alpha}_{1}, \tilde{\alpha}_{2}, \ldots, \tilde{\alpha}_{n^{2}}\right\}$. Let $\varphi: F_{n^{2}} \rightarrow \Gamma$ be the group homomorphism sending $\tilde{\alpha}_{i}$ to $\alpha_{i}$ for $1 \leq i \leq n^{2}$. Then $\varphi$ induces the following diagram:

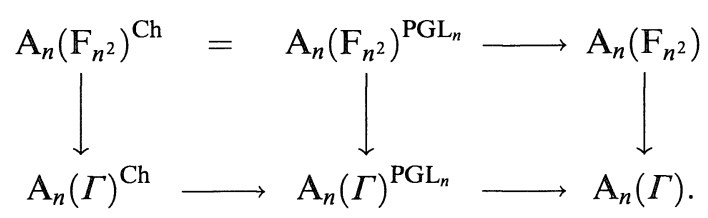

By Corollary 2.13 we have $\mathrm{A}_{n}\left(\mathrm{~F}_{n^{2}}\right)^{\mathrm{Ch}}=\mathrm{A}_{n}\left(\mathrm{~F}_{n^{2}}\right)^{\mathrm{PGL}_{n}}$. The discriminant $\Delta\left(\tilde{\alpha}_{1}, \ldots, \tilde{\alpha}_{n^{2}}\right)$ is sent to $\Delta\left(\alpha_{1}, \ldots, \alpha_{n^{2}}\right)$. So it suffices to show that $\Delta:=$ $\Delta\left(\tilde{\alpha}_{1}, \ldots, \tilde{\alpha}_{n^{2}}\right)$ is an element of $\mathrm{A}_{n}\left(\mathrm{~F}_{n^{2}}\right)^{\mathrm{PGL}_{n}}$. Moreover we only have to show that $\Delta$ is an element of $\left(\mathrm{A}_{n}\left(\mathrm{~F}_{n^{2}}\right) \otimes \mathbb{C}\right)^{\mathrm{PGL}_{n}}$, where $\mathbb{C}$ is the complex number field. By Remark 3.2 we see that $\Delta^{2} \in\left(\mathrm{A}_{n}\left(\mathrm{~F}_{n^{2}}\right) \otimes \mathrm{C}\right)^{\mathrm{PGL}_{n}}$. Since $\mathrm{PGL}_{n}$ has no nontrivial character, so we get $\Delta \in\left(\mathrm{A}_{n}\left(\mathrm{~F}_{n^{2}}\right) \otimes \mathbb{C}\right)^{\mathrm{PGL}_{n}}$. This completes the proof.

Now we define the absolutely irreducible representation part.

Definition 3.4. For $n^{2}$ elements $\alpha_{1}, \ldots, \alpha_{n^{2}}$ of a group $\Gamma$, we denote by $\operatorname{Rep}_{n}(\Gamma)_{\Delta\left(\alpha_{1}, \ldots, \alpha_{n^{2}}\right)}$ the open subscheme $\operatorname{Spec}\left(\mathrm{A}_{n}(\Gamma)_{\Delta\left(\alpha_{1}, \ldots, \alpha_{n^{2}}\right)}\right)$ of the representation variety $\operatorname{Rep}_{n}(\Gamma)=\operatorname{Spec} \mathrm{A}_{n}(\Gamma)$. We define the open subscheme $\operatorname{Rep}_{n}(\Gamma)_{\text {a.i.r. }}$ of $\operatorname{Rep}_{n}(\Gamma)$ by

$$
\operatorname{Rep}_{n}(\Gamma)_{\text {a.i.r. }}:=\bigcup_{\alpha_{1}, \alpha_{2}, \ldots, \alpha_{n^{2}} \in \Gamma} \operatorname{Rep}_{n}(\Gamma)_{\Delta\left(\alpha_{1}, \alpha_{2}, \ldots, \alpha_{n^{2}}\right)} .
$$


The open subscheme $\operatorname{Rep}_{n}(\Gamma)_{\text {a.i.r. }}$ is called the absolutely irreducible representation part or the a.i.r. part.

Recall that if $\rho: \Gamma \rightarrow \mathrm{GL}_{n}(k)$ is a representation with a field $k$, then $\rho$ is absolutely irreducible if and only if the subalgebra $k[\rho(\Gamma)]$ generated by $\rho(\Gamma)$ coincides with the full matrix ring $\mathbf{M}_{n}(k)$. Therefore the absolute irreducibility of $\rho$ is equivalent to the condition that there exist $n^{2}$ elements $\left\{\gamma_{i}\right\}_{1 \leq i \leq n^{2}}$ of $\Gamma$ such that $\left\{\rho\left(\gamma_{1}\right), \rho\left(\gamma_{2}\right), \ldots, \rho\left(\gamma_{n^{2}}\right)\right\}$ is a basis of $\mathbf{M}_{n}(k)$. Hence we easily see that $\operatorname{Rep}_{n}(\Gamma)_{\text {a.i.r. }}$ is the subfunctor of $\operatorname{Rep}_{n}(\Gamma)$ consisting of absolutely irreducible representations.

As in Definition 3.4 we define the a.i.r. parts of $\mathrm{Ch}_{n}(\Gamma)$ and $\mathrm{Ch}_{n}^{0}(\Gamma)$.

Definition 3.5. For elements $\alpha_{1}, \ldots, \alpha_{n^{2}}$ of a group $\Gamma$, we denote by $\mathrm{Ch}_{n}(\Gamma)_{\Delta\left(\alpha_{1}, \ldots, \alpha_{n^{2}}\right)}$ the open subscheme $\operatorname{Spec}\left(\left(\mathrm{A}_{n}(\Gamma)^{\mathrm{PGL}_{n}}\right)_{\Delta\left(\alpha_{1}, \ldots, \alpha_{n^{2}}\right)}\right)$ of $\mathrm{Ch}_{n}(\Gamma)$. Similarly we denote by $\mathrm{Ch}_{n}^{0}(\Gamma)_{\Delta\left(\alpha_{1}, \ldots, \alpha_{n^{2}}\right)}$ the open subscheme $\operatorname{Spec}\left(\left(\mathrm{A}_{n}(\Gamma)^{\mathrm{Ch}}\right)_{\Delta\left(\alpha_{1}, \ldots, \alpha_{n^{2}}\right)}\right)$ of $\mathrm{Ch}_{n}^{0}(\Gamma)$. We define the a.i.r. parts by

$$
\begin{aligned}
\mathrm{Ch}_{n}(\Gamma)_{\text {a.i.r. }}:=\bigcup_{\alpha_{1}, \alpha_{2}, \ldots, \alpha_{n^{2}} \in \Gamma} \mathrm{Ch}_{n}(\Gamma)_{\Delta\left(\alpha_{1}, \alpha_{2}, \ldots, \alpha_{n^{2}}\right)}, \\
\mathrm{Ch}_{n}^{0}(\Gamma)_{\text {a.i.r. }}:=\bigcup_{\alpha_{1}, \alpha_{2}, \ldots, \alpha_{n^{2}} \in \Gamma} \mathrm{Ch}_{n}^{0}(\Gamma)_{\Delta\left(\alpha_{1}, \alpha_{2}, \ldots, \alpha_{n^{2}}\right)} .
\end{aligned}
$$

Notation 3.6. Let $\alpha_{1}, \ldots, \alpha_{n^{2}}$ be elements of a group $\Gamma$ and let $\Delta$ be the discriminant $\Delta\left(\alpha_{1}, \ldots, \alpha_{n^{2}}\right)$. Then we get morphisms $\operatorname{Rep}_{n}(\Gamma)_{\Delta} \rightarrow \operatorname{Ch}_{n}(\Gamma)_{\Delta} \rightarrow$ $\mathrm{Ch}_{n}^{0}(\Gamma)_{\Delta}$ by restricting the morphisms $\pi_{n, \Gamma}: \operatorname{Rep}_{n}(\Gamma) \rightarrow \mathrm{Ch}_{n}(\Gamma) \rightarrow \mathrm{Ch}_{n}^{0}(\Gamma)$ in Notation 2.11; furthermore we get morphisms $\operatorname{Rep}_{n}(\Gamma)_{\text {a.i.r. }} \rightarrow \operatorname{Ch}_{n}(\Gamma)_{\text {a.i.r. }} \rightarrow$ $\mathrm{Ch}_{n}^{0}(\Gamma)_{\text {a.i.r. }}$. We shall denote by $\pi_{n, \Gamma, \Delta}$ and $\pi_{n, \Gamma \text {, air }}$ the morphisms $\operatorname{Rep}_{n}(\Gamma)_{\Delta} \rightarrow$ $\mathrm{Ch}_{n}^{0}(\Gamma)_{\Delta}$ and $\operatorname{Rep}_{n}(\Gamma)_{\text {a.i.r. }} \rightarrow \mathrm{Ch}_{n}^{0}(\Gamma)_{\text {a.i.r. }}$, respectively.

We shall later show that $\mathrm{Ch}_{n}(\Gamma)_{\text {a.i.r. }}=\mathrm{Ch}_{n}^{0}(\Gamma)_{\text {a.i.r. }}$ and that the morphism $\pi_{n, \Gamma \text {, air }}$ gives a universal geometric quotient by $\mathrm{PGL}_{n}$. In Section 6 we see that the scheme $\mathrm{Ch}_{n}(\Gamma)_{\text {a.i.r. }}\left(=\mathrm{Ch}_{n}^{0}(\Gamma)_{\text {a.i.r. }}\right)$ is the character variety, that is, the coarse moduli scheme of equivalence classes of absolutely irreducible representations.

The next proposition has no direct bearing on the proof of the main theorem but implies that the a.i.r. part of the representation variety is nonempty for each free group of rank $\geq 2$. Furthermore the following stronger result is also known: if a field $k$ is a transcendental extension of a prime field or if $k$ has characteristic 0 , then $\mathrm{SL}_{n}(k)$ has a $k$-dense subgroup which is isomorphic to the free group $F_{2}$ of rank 2 , and hence this subgroup $F_{2}$ possesses an absolutely irreducible representation arising from $\mathrm{SL}_{n}(k)$ (for proofs see [12]). Here we give an easy proof of the existence of absolutely irreducible representations for each free group of rank $\geq 2$. 
Proposition 3.7. Let $k$ be an arbitrary field. For the free group $\mathrm{F}_{m}$ of rank $m \geq 2$, there exists an absolutely irreducible representation $\rho: \mathrm{F}_{m} \rightarrow \mathrm{GL}_{n}(k)$ for each positive integer $n$. Furthermore we may assume that $\rho$ is a representation in $\mathrm{SL}_{n}(k)$.

Proof. First we shall prove the case $m=2$. Let $\{\alpha, \beta\}$ be a system of free generators of $\mathrm{F}_{2}$. We define the representation $\rho: \mathrm{F}_{2} \rightarrow \mathrm{GL}_{n}(k)$ by

$$
\rho(\alpha):=\left(\begin{array}{ccccc}
1 & 1 & 0 & \cdots & 0 \\
0 & 1 & 1 & \cdots & 0 \\
0 & 0 & 1 & \ddots & \vdots \\
\vdots & \vdots & \ddots & \ddots & 1 \\
0 & 0 & 0 & \cdots & 1
\end{array}\right), \quad \rho(\beta):=\left(\begin{array}{ccccc}
1 & 0 & 0 & \cdots & 0 \\
1 & 1 & 0 & \cdots & 0 \\
0 & 1 & 1 & \ddots & 0 \\
\vdots & \vdots & \ddots & \ddots & \vdots \\
0 & 0 & \cdots & 1 & 1
\end{array}\right) .
$$

We show that $\rho$ is absolutely irreducible. Let $\left\{e_{1}, \ldots, e_{n}\right\}$ be the canonical basis of $k^{\oplus n}$. Put $V_{i}:=\bigoplus_{j=1}^{i} k \cdot e_{j}$ and $W_{i}:=\bigoplus_{j=i}^{n} k \cdot e_{j}$. We can easily see that any $\rho(\alpha)$-invariant subspace of $V$ is either $\{0\}$ or some $V_{i}(i=1,2, \ldots, n)$. We can also check that any $\rho(\beta)$-invariant subspace is either $\{0\}$ or some $W_{i}$. Since there is no non-trivial subspace invariant under $\rho(\alpha)$ and $\rho(\beta)$, the representation $\rho$ is irreducible. We also see that it is absolutely irreducible.

In the case $\mathrm{F}_{m}$ with $m \geq 2$ we get an absolutely irreducible representation by composing a surjective morphism $\mathrm{F}_{m} \rightarrow \mathrm{F}_{2}$ and the absolutely irreducible representation above $\rho: \mathrm{F}_{2} \rightarrow \mathrm{GL}_{n}(k)$. The last statement follows from the construction of $\rho$.

We have thus proved that the absolutely irreducible representation part is non-empty for the case of the free group of rank $m \geq 2$.

Corollary 3.8. Let $k$ be a field. For the free group $\mathrm{F}_{m}$ of rank $m \geq 2$, there exist $n^{2}$ elements $\alpha_{1}, \ldots, \alpha_{n^{2}} \in \mathrm{F}_{m}$ such that $\Delta\left(\alpha_{1}, \ldots, \alpha_{n^{2}}\right) \neq 0$ in $\mathrm{A}_{n}\left(\mathrm{~F}_{m}\right) \otimes_{\mathbf{Z}} k . \quad$ In particular $\operatorname{Rep}_{n}\left(\mathrm{~F}_{m}\right)_{\text {a.i.r. }}$ is non-empty if $m \geq 2$.

\section{§4. Reconstruction of Matrices}

In this section we obtain an easy but useful result that any matrices can be reconstructed by their invariants (Theorem 4.1). As an application of this theorem, we can see that the ring $\mathrm{A}_{n}(\Gamma)_{\Delta}^{\mathrm{Ch}}$ is a finitely generated algebra over $\mathbb{Z}$ if $\Gamma$ is a finitely generated group (Corollary 4.4). Moreover we shall apply Theorem 4.1 to the proofs of Theorem 5.1 in $\S 5$ and Theorem 6.3 in $\S 6$.

Theorem 4.1 (Reconstruction of Matrices). Let $R$ be a commutative ring, and let $A_{1}, A_{2}, \ldots, A_{n^{2}}$ be $n^{2}$ elements of $\mathrm{M}_{n}(R)$ such that the discriminant $\Delta\left(A_{1}, A_{2}, \ldots, A_{n^{2}}\right)$ is an invertible element of $R$. Then for any $X \in \mathrm{M}_{n}(R)$ we 
have

where

$$
X=\left(A_{1}, A_{2}, \ldots, A_{n^{2}}\right) T\left(\begin{array}{c}
\operatorname{tr}\left(A_{1} X\right) \\
\operatorname{tr}\left(A_{2} X\right) \\
\vdots \\
\operatorname{tr}\left(A_{n^{2}} X\right)
\end{array}\right)
$$

$$
T=\left(\begin{array}{cccc}
\operatorname{tr}\left(A_{1} A_{1}\right) & \operatorname{tr}\left(A_{1} A_{2}\right) & \ldots & \operatorname{tr}\left(A_{1} A_{n^{2}}\right) \\
\operatorname{tr}\left(A_{2} A_{1}\right) & \operatorname{tr}\left(A_{2} A_{2}\right) & \ldots & \operatorname{tr}\left(A_{2} A_{n^{2}}\right) \\
\vdots & \vdots & \ldots & \vdots \\
\operatorname{tr}\left(A_{n^{2}} A_{1}\right) & \operatorname{tr}\left(A_{n^{2}} A_{2}\right) & \ldots & \operatorname{tr}\left(A_{n^{2}} A_{n^{2}}\right)
\end{array}\right)^{-1} \in \mathrm{GL}_{n^{2}}(R)
$$

Proof. Since $\Delta\left(A_{1}, \ldots, A_{n^{2}}\right) \in R^{\times}$, the set $\left\{A_{1}, \ldots, A_{n^{2}}\right\}$ is a free basis of $\mathrm{M}_{n}(R)$ over $R$ (cf. Remark 3.2). Recall that the bilinear form

$$
\begin{aligned}
\operatorname{tr}(\cdot): \mathrm{M}_{n}(R) \times \mathrm{M}_{n}(R) & \rightarrow R \\
(X, Y) & \mapsto \operatorname{tr}(X Y)
\end{aligned}
$$

is a perfect pairing. The equality easily follows from the fact that $\left(A_{1}, \ldots, A_{n^{2}}\right) T$ is the dual basis of $\left(A_{1}, \ldots, A_{n^{2}}\right)$ with respect to $\operatorname{tr}(\cdot)$.

Corollary 4.2. Under the same situation as Theorem 4.1, assume that $X, Y \in \mathrm{M}_{n}(R)$. Then we obtain the equality

$$
\operatorname{tr}(X Y)=\left(\operatorname{tr}\left(A_{1} X\right), \ldots, \operatorname{tr}\left(A_{n^{2}} X\right)\right) T\left(\begin{array}{c}
\operatorname{tr}\left(A_{1} Y\right) \\
\vdots \\
\operatorname{tr}\left(A_{n^{2}} Y\right)
\end{array}\right) .
$$

Proof. Using Theorem 4.1, we have

$$
Y=\left(A_{1}, \ldots, A_{n^{2}}\right) T\left(\begin{array}{c}
\operatorname{tr}\left(A_{1} Y\right) \\
\vdots \\
\operatorname{tr}\left(A_{n^{2}} Y\right)
\end{array}\right) .
$$

Multiply $X$ from the left and take the traces of the both sides.

We shall prove that $\mathrm{A}_{n}(\Gamma)_{\Delta}^{\mathrm{Ch}}$ is a finitely generated algebra over $\mathbf{Z}$ if $\Gamma$ is a finitely generated group. This fact can be also verified from Donkin's Theorem (Theorem 2.12) and the invariant theory, but the proof of this paper gives explicit generators of $\mathrm{A}_{n}(\Gamma)_{\Delta}^{\mathrm{Ch}}$.

Theorem 4.3. Let $\alpha_{1}, \ldots, \alpha_{n^{2}}$ be elements of the free group $\mathrm{F}_{m}$ of rank $m$ and let $\Delta$ be the discriminant $\Delta\left(\alpha_{1}, \ldots, \alpha_{n^{2}}\right)$. Then $\mathrm{A}_{n}\left(\mathrm{~F}_{m}\right)_{\Delta}^{\text {Ch }}$ is a finitely generated algebra over $\mathbf{Z}$. 
Proof. Let $f_{i}\left(x_{1}, \ldots, x_{n^{2}}\right)$ denote $c_{i}\left(x_{1} \sigma_{\mathrm{F}_{m}}\left(\alpha_{1}\right)+\cdots+x_{n^{2}} \sigma_{\mathrm{F}_{m}}\left(\alpha_{n^{2}}\right)\right)$, where $\sigma_{\mathrm{F}_{m}}$ is the universal representation of degree $n$ for $\mathrm{F}_{m}$. We easily see that $f_{i} \in \mathbf{A}_{n}\left(\mathrm{~F}_{m}\right)^{\mathrm{PGL}_{n}}\left[x_{1}, \ldots, x_{n^{2}}\right]=\mathbf{A}_{n}\left(\mathrm{~F}_{m}\right)^{\mathrm{Ch}}\left[x_{1}, \ldots, x_{n^{2}}\right]$. By Theorem 4.1 we have

$$
\sigma_{\mathrm{F}_{m}}(\gamma)=\left(\sigma_{\mathrm{F}_{m}}\left(\alpha_{1}\right), \ldots, \sigma_{\mathrm{F}_{m}}\left(\alpha_{n^{2}}\right)\right) T\left(\begin{array}{c}
s\left(\alpha_{1} \gamma\right) \\
\vdots \\
s\left(\alpha_{n^{2}} \gamma\right)
\end{array}\right) \quad \text { for each } \gamma \in \Gamma,
$$

where $T=\left(s\left(\alpha_{i} \alpha_{j}\right)\right)_{1 \leq i, j \leq n^{2}}^{-1}$. Here the symbol $s(\cdot)$ is as in Notation 2.9. Therefore there exist $n^{2}$ polynomials $g_{1}, \ldots, g_{n^{2}}$ in the entries of $T$ and $\left\{s(\delta) \mid \delta \in \mathrm{F}_{m}\right\}$ such that

$$
c_{i}(\gamma)=c_{i}\left(\sigma_{\mathrm{F}_{m}}(\gamma)\right)=f_{i}\left(g_{1}, \ldots, g_{n^{2}}\right) .
$$

We have thus shown that $\mathrm{A}_{n}\left(\mathrm{~F}_{m}\right)_{\Delta}^{\mathrm{Ch}}$ is generated by the entries of $T$, the coefficients of $f_{i}(1 \leq i \leq n)$ and $\left\{s(\delta) \mid \delta \in \mathrm{F}_{m}\right\}$ over $\mathbb{Z}$.

We take a system of free generators $\left\{\beta_{1}, \ldots, \beta_{m}\right\}$ of $\mathrm{F}_{m}$. For $\gamma \in \Gamma$ we define the length of $\gamma$ with respect to $\left\{\beta_{1}, \ldots, \beta_{m}\right\}$ by

$$
\text { length }(\gamma):=\min \left\{p \mid \gamma=\beta_{i_{1}}^{\varepsilon_{1}} \cdots \beta_{i_{p}}^{\varepsilon_{p}}, \varepsilon_{i}= \pm 1\right\} .
$$

Put $\ell:=\max \left\{\operatorname{length}\left(\alpha_{i}\right) \mid 1 \leq i \leq n^{2}\right\}+1$. The theorem follows from the claim that $\mathrm{A}_{n}\left(\mathrm{~F}_{m}\right)_{\Delta}^{\mathrm{Ch}}$ is generated by the entries of $T$, the coefficients of $f_{i}$, and $\{s(\delta) \mid$ length $(\delta)<2 \ell\}$. For the proof of this claim, we only have to prove that $s(\gamma)$ is contained in the subalgebra generated by these elements if length $(\gamma) \geq 2 \ell$.

Assume that length $(\gamma) \geq 2 \ell$. Then we can choose $\gamma_{1}, \gamma_{2} \in \mathbb{F}_{m}$ such that $\gamma=\gamma_{1} \gamma_{2}$, length $\left(\gamma_{1}\right) \geq \ell$, length $\left(\gamma_{2}\right) \geq \ell$, and length $\left(\gamma_{1}\right)+$ length $\left(\gamma_{2}\right)=\operatorname{length}(\gamma)$. By Corollary 4.2 we have

$$
s(\gamma)=s\left(\gamma_{1} \gamma_{2}\right)=\left(s\left(\alpha_{1} \gamma_{1}\right), \ldots, s\left(\alpha_{n^{2}} \gamma_{1}\right)\right) T\left(\begin{array}{c}
s\left(\alpha_{1} \gamma_{2}\right) \\
\vdots \\
s\left(\alpha_{n^{2}} \gamma_{2}\right)
\end{array}\right) .
$$

Since length $\left(\alpha_{i} \gamma_{1}\right)<$ length $(\gamma)$ and length $\left(\alpha_{i} \gamma_{2}\right)<$ length $(\gamma)$, we can show that $s(\gamma)$ is contained in the subalgebra generated by the entries of $T$ and $\{s(\delta) \mid$ length $(\delta)<2 \ell\}$ by induction. This completes the proof.

Corollary 4.4. Let $\Gamma$ be a finitely generated group. For $\alpha_{1}, \alpha_{2}, \ldots, \alpha_{n^{2}} \in \Gamma$ we denote $\Delta\left(\alpha_{1}, \alpha_{2}, \ldots, \alpha_{n^{2}}\right)$ by $\Delta$. Then $\mathrm{A}_{n}(\Gamma)_{\Delta}^{\mathrm{Ch}}$ is a finitely generated algebra over $\mathbf{Z}$.

Proof. There exists a surjective group homomorphism $\varphi: \mathbb{F}_{m} \rightarrow \Gamma$. Take $\tilde{\alpha}_{i} \in \mathrm{F}_{m}$ such that $\varphi\left(\tilde{\alpha}_{i}\right)=\alpha_{i}$ for $1 \leq i \leq n^{2}$. Then $\varphi$ induces a surjective homomorphism $\mathrm{A}_{n}\left(\mathrm{~F}_{m}\right)_{\Delta\left(\tilde{\alpha}_{1}, \ldots, \tilde{\alpha}_{n^{2}}\right)}^{\mathrm{Ch}} \rightarrow \mathrm{A}_{n}(\Gamma)_{\Delta}^{\mathrm{Ch}}$. The statement follows from Theorem 4.3. 
The following corollary shall be used in the next section.

Corollary 4.5. Let $\alpha_{1}, \alpha_{2}, \ldots, \alpha_{n^{2}}$ be elements of a group $\Gamma$ and let $\Delta$ be the discriminant $\Delta\left(\alpha_{1}, \alpha_{2}, \ldots, \alpha_{n^{2}}\right)$. Suppose that $\mathrm{F}_{n^{2}}$ is the free group with a system of free generators $\left\{\tilde{\alpha}_{1}, \ldots, \tilde{\alpha}_{n^{2}}\right\}$. Let $\phi: \mathbf{A}_{n}\left(\mathrm{~F}_{n^{2}}\right)_{\Delta\left(\tilde{\alpha}_{1}, \ldots, \tilde{\alpha}_{n^{2}}\right)}^{\mathrm{Ch}} \rightarrow \mathrm{A}_{n}(\Gamma)_{\Delta}^{\mathrm{Ch}}$ denote the $\mathbb{Z}$-algebra homomorphism induced by the group homomorphism $\mathrm{F}_{n^{2}} \rightarrow \Gamma$ sending $\tilde{\alpha}_{i}$ to $\alpha_{i}$ for $1 \leq i \leq n^{2}$. Then the ring $\mathrm{A}_{n}(\Gamma)_{\Delta}^{\mathrm{Ch}}$ is generated by $\{s(\gamma) \mid \gamma \in \Gamma\}$ and $\operatorname{Im} \phi$ as a Z-algebra.

Proof. We denote by $\mathscr{B}$ the subalgebra of $\mathrm{A}_{n}(\Gamma)_{\Delta}^{\mathrm{Ch}}$ generated by $\{s(\gamma) \mid \gamma \in \Gamma\}$ and $\operatorname{Im} \phi$ over $\mathbf{Z}$. It suffices to show that any $c_{i}(\gamma)$ is contained in $\mathscr{B}$. Put $f_{i}\left(x_{1}, \ldots, x_{n^{2}}\right):=c_{i}\left(x_{1} \sigma_{\mathrm{F}_{n^{2}}}\left(\tilde{\alpha}_{1}\right)+\cdots+x_{n^{2}} \sigma_{\mathrm{F}_{n^{2}}}\left(\tilde{\alpha}_{n^{2}}\right)\right) \in \mathrm{A}_{n}\left(\mathrm{~F}_{n^{2}}\right)_{\tilde{\Delta}}^{\mathrm{Ch}}\left[x_{1}, \ldots, x_{n^{2}}\right]$, where $\tilde{\Delta}:=\Delta\left(\tilde{\alpha}_{1}, \ldots, \tilde{\alpha}_{n^{2}}\right)$. Sending the coefficients of $f_{i}$ by $\phi$, we have $f_{i}^{\phi}\left(x_{1}, \ldots, x_{n^{2}}\right)=c_{i}\left(x_{1} \sigma_{\Gamma}\left(\alpha_{1}\right)+\cdots+x_{n^{2}} \sigma_{\Gamma}\left(\alpha_{n^{2}}\right)\right) \in \mathrm{A}_{n}(\Gamma)_{\Delta}^{\mathrm{Ch}}\left[x_{1}, \ldots, x_{n^{2}}\right] . \quad$ Вy Theorem 4.1 we have

$$
\sigma_{\Gamma}(\gamma)=\left(\sigma_{\Gamma}\left(\alpha_{1}\right), \ldots, \sigma_{\Gamma}\left(\alpha_{n^{2}}\right)\right) T\left(\begin{array}{c}
s\left(\alpha_{1} \gamma\right) \\
\vdots \\
s\left(\alpha_{n^{2}} \gamma\right)
\end{array}\right) \quad \text { for each } \gamma \in \Gamma
$$

where $T=\left(s\left(\alpha_{i} \alpha_{j}\right)\right)_{1 \leq i, j \leq n}^{-1}$. Therefore each $c_{i}(\gamma)$ is expressed as a polynomial in $\{s(\delta) \mid \delta \in \Gamma\}$, the coefficients of $f_{i}^{\phi}$ and the entries of $T$. Since the coefficients of $f_{i}^{\phi}$ and the entries of $T$ are contained in $\operatorname{Im} \phi$, any $c_{i}(\gamma)$ is contained in $\mathscr{B}$.

\section{§5. Reconstruction of Representations}

For any group $\Gamma$ and any discriminant $\Delta$, we shall see later that the morphism $\pi_{n, \Gamma, \Delta}: \operatorname{Rep}_{n}(\Gamma)_{\Delta} \rightarrow \mathrm{Ch}_{n}^{0}(\Gamma)_{\Delta}$ gives a universal geometric quotient by $\mathrm{PGL}_{n}(\S 6)$. For each degree $n$ there exists a special one among these morphisms $\pi_{n, \Gamma, \Delta}$. Let $\mathrm{F}_{n^{2}}=\left\langle\tilde{\alpha}_{1}, \ldots, \tilde{\alpha}_{n^{2}}\right\rangle$ be the free group of rank $n^{2}$. Set $\tilde{\Delta}:=\Delta\left(\tilde{\alpha}_{1}, \ldots, \tilde{\alpha}_{n^{2}}\right)$. The special morphism is $\pi_{n, \mathrm{~F}_{n^{2}}, \tilde{\Delta}}: \operatorname{Rep}_{n}\left(\mathrm{~F}_{n^{2}}\right)_{\tilde{\Delta}} \rightarrow \operatorname{Ch}_{n}^{0}\left(\mathrm{~F}_{n^{2}}\right)_{\tilde{\Delta}}$, which is the so-called "prototype" of degree $n$. In this section we show that for any group $\Gamma$ and any discriminant $\Delta$, the morphism $\pi_{n, \Gamma, \Delta}: \operatorname{Rep}_{n}(\Gamma)_{\Delta} \rightarrow$ $\mathrm{Ch}_{n}^{0}(\Gamma)_{\Delta}$ is obtained by the base change of the "prototype" (Theorem 5.1). This result suggests that we may reduce the arbitrary group case to the free group case for proving that the morphism $\pi_{n, \Gamma, \Delta}$ gives a universal geometric quotient.

Theorem 5.1 (Reconstruction of Representations). Let $\alpha_{1}, \ldots, \alpha_{n^{2}}$ be elements of a group $\Gamma$ and $\Delta$ the discriminant $\Delta\left(\alpha_{1}, \ldots, \alpha_{n^{2}}\right)$. Let $\mathrm{F}_{n^{2}}$ be the free group with a system of free generators $\left\{\tilde{\alpha}_{1}, \ldots, \tilde{\alpha}_{n^{2}}\right\}$. We denote by $\varphi: \mathrm{F}_{n^{2}} \rightarrow \Gamma$ the group homomorphism sending $\tilde{\alpha}_{i}$ to $\alpha_{i}$ for $1 \leq i \leq n^{2}$. Then the homo- 
morphism $\varphi$ induces the following diagram which is a fiber product:

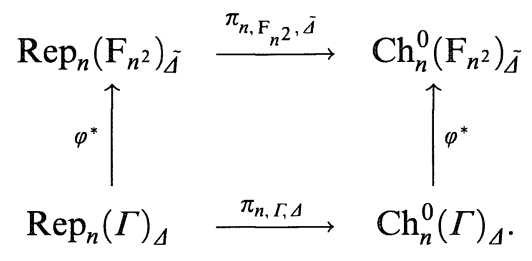

This theorem follows from the following lemma:

Lemma 5.2. Let us consider the same situation as Theorem 5.1. For any commutative ring $R$ and any commutative diagram

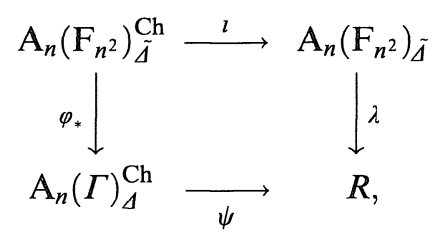

there exists a unique ring homomorphism $\tilde{\sigma}: \mathrm{A}_{n}(\Gamma)_{\Delta} \rightarrow R$ such that the following diagram is commutative:

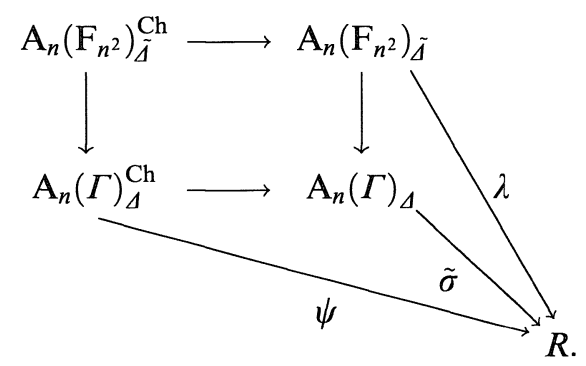

Proof of Lemma 5.2. First we prove the uniqueness of the ring homomorphism $\tilde{\sigma}$. By Theorem 4.1 we see that if $\tilde{\sigma}$ exists, then the corresponding representation $\sigma: \Gamma \rightarrow \mathrm{GL}_{n}(R)$ is given by

$$
\sigma(\gamma)=\left(\sigma\left(\alpha_{1}\right), \ldots, \sigma\left(\alpha_{n^{2}}\right)\right)\left(\operatorname{tr}\left(\sigma\left(\alpha_{i} \alpha_{j}\right)\right)\right)_{1 \leq i, j \leq n^{2}}^{-1}\left(\begin{array}{c}
\operatorname{tr}\left(\sigma\left(\alpha_{1} \gamma\right)\right) \\
\vdots \\
\operatorname{tr}\left(\sigma\left(\alpha_{n^{2}} \gamma\right)\right)
\end{array}\right)
$$

Let $\rho: \mathrm{F}_{n^{2}} \rightarrow \mathrm{GL}_{n}(R)$ be the representation associated to $\lambda$. We obtain $\left(\rho\left(\tilde{\alpha}_{1}\right), \ldots, \rho\left(\tilde{\alpha}_{n^{2}}\right)\right)=\left(\sigma\left(\alpha_{1}\right), \ldots, \sigma\left(\alpha_{n^{2}}\right)\right)$ and $\left(\operatorname{tr}\left(\sigma\left(\alpha_{i} \alpha_{j}\right)\right)\right)_{1 \leq i, j \leq n^{2}}^{-1}=$ $\left(\psi\left(s\left(\alpha_{i} \alpha_{j}\right)\right)\right)_{1 \leq i, j \leq n^{2}}^{-1}$ by the commutativity of the diagram. Put $T=$ $\left(\psi\left(s\left(\alpha_{i} \alpha_{j}\right)\right)\right)_{1 \leq i, j \leq n^{2}}^{-1}$ and $\left(A_{1}, \ldots, A_{n^{2}}\right)=\left(\rho\left(\tilde{\alpha}_{1}\right), \ldots, \rho\left(\tilde{\alpha}_{n^{2}}\right)\right)$. Then we have 


$$
\sigma(\gamma)=\left(A_{1}, \ldots, A_{n^{2}}\right) T\left(\begin{array}{c}
\psi\left(s\left(\alpha_{1} \gamma\right)\right) \\
\vdots \\
\psi\left(s\left(\alpha_{n^{2}} \gamma\right)\right)
\end{array}\right) \quad \text { for each } \gamma \in \Gamma .
$$

This implies the uniqueness of $\tilde{\sigma}$.

For proving the existence of $\tilde{\sigma}$, it suffices to show that the map $\sigma: \Gamma \rightarrow \mathrm{M}_{n}(R)$ defined by (1) is a representation such that the diagram is commutative. After the following seven claims, we complete the proof.

Claim 1. The following equality holds:

$$
\left(\sigma\left(\alpha_{1}\right), \ldots, \sigma\left(\alpha_{n^{2}}\right)\right)=\left(A_{1}, \ldots, A_{n^{2}}\right) .
$$

Proof. The statement is verified from the following:

$$
\begin{aligned}
\text { L.H.S. } & =\left(A_{1}, \ldots, A_{n^{2}}\right) T\left(\begin{array}{ccc}
\psi\left(s\left(\alpha_{1} \alpha_{1}\right)\right) & \cdots & \psi\left(s\left(\alpha_{1} \alpha_{n^{2}}\right)\right) \\
\vdots & \psi\left(s\left(\alpha_{i} \alpha_{j}\right)\right) & \vdots \\
\psi\left(s\left(\alpha_{n^{2}} \alpha_{1}\right)\right) & \cdots & \psi\left(s\left(\alpha_{n^{2}} \alpha_{n^{2}}\right)\right)
\end{array}\right) \\
& =\left(A_{1}, \ldots, A_{n^{2}}\right) .
\end{aligned}
$$

Claim 2. For each $\gamma \in \Gamma$, we have

$$
\left(\begin{array}{c}
\operatorname{tr}\left(A_{1} \sigma(\gamma)\right) \\
\vdots \\
\operatorname{tr}\left(A_{n^{2}} \sigma(\gamma)\right)
\end{array}\right)=\left(\begin{array}{c}
\psi\left(s\left(\alpha_{1} \gamma\right)\right) \\
\vdots \\
\psi\left(s\left(\alpha_{n^{2}} \gamma\right)\right)
\end{array}\right)
$$

Proof. Note that $T=\left(\operatorname{tr}\left(A_{i} A_{j}\right)\right)^{-1}$. Multiply the both sides of (1) by $A_{1}, \ldots, A_{n^{2}}$ from the left and take the traces. Then we obtain the following:

$$
\begin{aligned}
\left(\begin{array}{c}
\operatorname{tr}\left(A_{1} \sigma(\gamma)\right) \\
\vdots \\
\operatorname{tr}\left(A_{n^{2}} \sigma(\gamma)\right)
\end{array}\right) & =\left(\begin{array}{ccc}
\operatorname{tr}\left(A_{1} A_{1}\right) & \cdots & \operatorname{tr}\left(A_{1} A_{n^{2}}\right) \\
\vdots & \operatorname{tr}\left(A_{i} A_{j}\right) & \vdots \\
\operatorname{tr}\left(A_{n^{2}} A_{1}\right) & \ldots & \operatorname{tr}\left(A_{n^{2}} A_{n^{2}}\right)
\end{array}\right) T\left(\begin{array}{c}
\psi\left(s\left(\alpha_{1} \gamma\right)\right) \\
\vdots \\
\psi\left(s\left(\alpha_{n^{2}} \gamma\right)\right)
\end{array}\right) \\
& =\left(\begin{array}{c}
\psi\left(s\left(\alpha_{1} \gamma\right)\right) \\
\vdots \\
\psi\left(s\left(\alpha_{n^{2}} \gamma\right)\right)
\end{array}\right) .
\end{aligned}
$$

Claim 3. For $\gamma \in \Gamma$ and $1 \leq i, j \leq n^{2}$, we have

$$
\operatorname{tr}\left(A_{i} \sigma(\gamma) A_{j}\right)=\psi\left(s\left(\alpha_{i} \gamma \alpha_{j}\right)\right)
$$

Proof. Multiply the both sides of (1) by $A_{i}$ from the left and by $A_{1}, \ldots, A_{n^{2}}$ from the right. Then we obtain 


$$
\left(\begin{array}{c}
A_{i} \sigma(\gamma) A_{1} \\
A_{i} \sigma(\gamma) A_{2} \\
\vdots \\
A_{i} \sigma(\gamma) A_{n^{2}}
\end{array}\right)=\left(\begin{array}{cccc}
A_{i} A_{1} A_{1} & A_{i} A_{2} A_{1} & \cdots & A_{i} A_{n^{2}} A_{1} \\
A_{i} A_{1} A_{2} & A_{i} A_{2} A_{2} & \cdots & A_{i} A_{n^{2}} A_{2} \\
\vdots & \vdots & \cdots & \vdots \\
A_{i} A_{1} A_{n^{2}} & A_{i} A_{2} A_{n^{2}} & \cdots & A_{i} A_{n^{2}} A_{n^{2}}
\end{array}\right) T\left(\begin{array}{c}
\psi\left(s\left(\alpha_{1} \gamma\right)\right) \\
\psi\left(s\left(\alpha_{2} \gamma\right)\right) \\
\vdots \\
\psi\left(s\left(\alpha_{n^{2}} \gamma\right)\right)
\end{array}\right)
$$

Taking the traces of the both sides, we get

$$
\begin{aligned}
\left(\begin{array}{c}
\operatorname{tr}\left(A_{i} \sigma(\gamma) A_{1}\right) \\
\vdots \\
\operatorname{tr}\left(A_{i} \sigma(\gamma) A_{n^{2}}\right)
\end{array}\right) & =\left(\begin{array}{ccc}
\operatorname{tr}\left(A_{i} A_{1} A_{1}\right) & \cdots & \operatorname{tr}\left(A_{i} A_{n^{2}} A_{1}\right) \\
\vdots & \cdots & \vdots \\
\operatorname{tr}\left(A_{i} A_{1} A_{n^{2}}\right) & \cdots & \operatorname{tr}\left(A_{i} A_{n^{2}} A_{n^{2}}\right)
\end{array}\right) T\left(\begin{array}{c}
\psi\left(s\left(\alpha_{1} \gamma\right)\right) \\
\psi\left(s\left(\alpha_{2} \gamma\right)\right) \\
\vdots \\
\psi\left(s\left(\alpha_{n^{2}} \gamma\right)\right)
\end{array}\right) \\
& =\left(\begin{array}{ccc}
\psi\left(s\left(\alpha_{i} \alpha_{1} \alpha_{1}\right)\right) & \cdots & \psi\left(s\left(\alpha_{i} \alpha_{n^{2}} \alpha_{1}\right)\right) \\
\vdots & \cdots & \vdots \\
\psi\left(s\left(\alpha_{1} \gamma\right)\right) \\
\psi\left(s\left(\alpha_{2} \gamma\right)\right) \\
\vdots \\
\psi\left(s\left(\alpha_{n^{2}} \gamma\right)\right)
\end{array}\right) \\
& =\left(\begin{array}{c}
\psi\left(s\left(\alpha_{i} \gamma \alpha_{n^{2}}\right)\right) \\
\psi\left(s\left(\alpha_{i} \gamma \alpha_{2}\right)\right) \\
\vdots \\
\psi\left(s\left(\alpha_{i} \gamma \alpha_{n^{2}}\right)\right)
\end{array}\right)
\end{aligned}
$$

Remark that the last equality is obtained by taking the traces of the both sides of the following and by applying $\psi$ :

$$
\begin{aligned}
\left(\begin{array}{c}
\sigma_{\Gamma}\left(\alpha_{i} \gamma \alpha_{1}\right) \\
\vdots \\
\sigma_{\Gamma}\left(\alpha_{i} \gamma \alpha_{n^{2}}\right)
\end{array}\right)= & \left(\begin{array}{ccc}
\sigma_{\Gamma}\left(\alpha_{i} \alpha_{1} \alpha_{1}\right) & \cdots & \sigma_{\Gamma}\left(\alpha_{i} \alpha_{n^{2}} \alpha_{1}\right) \\
\vdots & \cdots & \vdots \\
\sigma_{\Gamma}\left(\alpha_{i} \alpha_{1} \alpha_{n^{2}}\right) & \cdots & \sigma_{\Gamma}\left(\alpha_{i} \alpha_{n^{2}} \alpha_{n^{2}}\right)
\end{array}\right) \\
& \times\left(s\left(\alpha_{k} \alpha_{\ell}\right)\right)_{1 \leq k, \ell \leq n^{2}}^{-1}\left(\begin{array}{c}
s\left(\alpha_{1} \gamma\right) \\
s\left(\alpha_{2} \gamma\right) \\
\vdots \\
s\left(\alpha_{n^{2}} \gamma\right)
\end{array}\right),
\end{aligned}
$$

where $\sigma_{\Gamma}$ is the universal representation for $\Gamma$. This completes the proof of the claim. 
Claim 4. For $\gamma, \delta \in \Gamma$, we have

$$
\sigma(\gamma) \sigma(\delta)=\sigma(\gamma \delta)
$$

Proof. Put

$$
C:=\left(\begin{array}{c}
\operatorname{tr}\left(A_{1} \sigma(\gamma) \sigma(\delta)\right) \\
\cdots \\
\operatorname{tr}\left(A_{n^{2}} \sigma(\gamma) \sigma(\delta)\right)
\end{array}\right)
$$

From the definition of $\sigma(\delta)$ we get

$$
\left(\begin{array}{c}
A_{1} \sigma(\gamma) \sigma(\delta) \\
\vdots \\
A_{n^{2}} \sigma(\gamma) \sigma(\delta)
\end{array}\right)=\left(\begin{array}{ccc}
A_{1} \sigma(\gamma) A_{1} & \ldots & A_{1} \sigma(\gamma) A_{n^{2}} \\
\vdots & A_{i} \sigma(\gamma) A_{j} & \vdots \\
A_{n^{2}} \sigma(\gamma) A_{1} & \ldots & A_{n^{2}} \sigma(\gamma) A_{n^{2}}
\end{array}\right) T\left(\begin{array}{c}
\psi\left(s\left(\alpha_{1} \delta\right)\right) \\
\vdots \\
\psi\left(s\left(\alpha_{n^{2}} \delta\right)\right)
\end{array}\right)
$$

Taking the traces of the both sides and using Claim 3, we obtain

$$
\begin{aligned}
C & =\left(\begin{array}{ccc}
\operatorname{tr}\left(A_{1} \sigma(\gamma) A_{1}\right) & \ldots & \operatorname{tr}\left(A_{1} \sigma(\gamma) A_{n^{2}}\right) \\
\vdots & \operatorname{tr}\left(A_{i} \sigma(\gamma) A_{j}\right) & \vdots \\
\operatorname{tr}\left(A_{n^{2}} \sigma(\gamma) A_{1}\right) & \ldots & \operatorname{tr}\left(A_{n^{2}} \sigma(\gamma) A_{n^{2}}\right)
\end{array}\right) T\left(\begin{array}{c}
\psi\left(s\left(\alpha_{1} \delta\right)\right) \\
\vdots \\
\psi\left(s\left(\alpha_{n^{2}} \delta\right)\right)
\end{array}\right) \\
& =\left(\begin{array}{ccc}
\psi\left(s\left(\alpha_{1} \gamma \alpha_{1}\right)\right) & \ldots & \psi\left(s\left(\alpha_{1} \gamma \alpha_{n^{2}}\right)\right) \\
\vdots & \psi\left(s\left(\alpha_{i} \gamma \alpha_{j}\right)\right) & \vdots \\
\psi\left(s\left(\alpha_{n^{2}} \gamma \alpha_{1}\right)\right) & \ldots & \psi\left(s\left(\alpha_{n^{2}} \gamma \alpha_{n^{2}}\right)\right)
\end{array}\right) T\left(\begin{array}{c}
\psi\left(s\left(\alpha_{1} \delta\right)\right) \\
\vdots \\
\psi\left(s\left(\alpha_{n^{2}} \delta\right)\right)
\end{array}\right) .
\end{aligned}
$$

Considering the universal representation $\sigma_{\Gamma}$ for $\Gamma$, we obtain

By Claim 2, we have

$$
C=\left(\begin{array}{c}
\psi\left(s\left(\alpha_{1} \gamma \delta\right)\right) \\
\vdots \\
\psi\left(s\left(\alpha_{n^{2}} \gamma \delta\right)\right)
\end{array}\right)
$$

$$
\left(\begin{array}{c}
\psi\left(s\left(\alpha_{1} \gamma \delta\right)\right) \\
\vdots \\
\psi\left(s\left(\alpha_{n^{2}} \gamma \delta\right)\right)
\end{array}\right)=\left(\begin{array}{c}
\operatorname{tr}\left(A_{1} \sigma(\gamma \delta)\right) \\
\vdots \\
\operatorname{tr}\left(A_{n^{2}} \sigma(\gamma \delta)\right)
\end{array}\right)
$$

Therefore we obtain the equality

$$
\left(\begin{array}{c}
\operatorname{tr}\left(A_{1} \sigma(\gamma) \sigma(\delta)\right) \\
\vdots \\
\operatorname{tr}\left(A_{n^{2}} \sigma(\gamma) \sigma(\delta)\right)
\end{array}\right)=\left(\begin{array}{c}
\operatorname{tr}\left(A_{1} \sigma(\gamma \delta)\right) \\
\vdots \\
\operatorname{tr}\left(A_{n^{2}} \sigma(\gamma \delta)\right)
\end{array}\right)
$$

Since $\Delta\left(A_{1}, \ldots, A_{n^{2}}\right) \in R^{\times}$, we conclude that $\sigma(\gamma) \sigma(\delta)=\sigma(\gamma \delta)$. 
Claim 5. The following equality holds:

$$
\sigma(e)=I_{n}
$$

Proof. By the definition we have

$$
\sigma(e)=\left(A_{1}, \ldots, A_{n^{2}}\right) T\left(\begin{array}{c}
\psi\left(s\left(\alpha_{1}\right)\right) \\
\vdots \\
\psi\left(s\left(\alpha_{n^{2}}\right)\right)
\end{array}\right) .
$$

Using Theorem 4.1, we also obtain

$$
I_{n}=\left(A_{1}, \ldots, A_{n^{2}}\right) T\left(\begin{array}{c}
\operatorname{tr}\left(A_{1}\right) \\
\vdots \\
\operatorname{tr}\left(A_{n^{2}}\right)
\end{array}\right) .
$$

This implies that the statement is true.

By the five claims above, we see that the map $\sigma: \Gamma \rightarrow \mathrm{GL}_{n}(R)$ is a representation.

Claim 6. For $\gamma \in \Gamma$, we have

$$
\operatorname{tr}(\sigma(\gamma))=\psi(s(\gamma))
$$

Proof. By Claim 2 we have

$$
\operatorname{tr}\left(A_{i} \sigma(\gamma)\right)=\psi\left(s\left(\alpha_{i} \gamma\right)\right) .
$$

Substituting $\alpha_{i}^{-1} \gamma$ for $\gamma$, we can prove Claim 6. For the semigroup (or monoid) case we can also prove Claim 6 by the definition of $\sigma(\gamma)$ and Corollary 4.2.

Claim 7. The following diagram commutes:

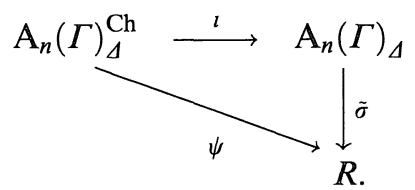

Proof. We show that two ring homomorphisms $\psi$ and $\tilde{\sigma} \circ l$ coincide. By Corollary 4.5 the ring $\mathrm{A}_{n}(\Gamma)_{\Delta}^{\mathrm{Ch}}$ is generated by $\{s(\gamma) \mid \gamma \in \Gamma\}$ and $\operatorname{Im}(\phi)$ over $\mathbb{Z}$, where $\phi\left(=\varphi_{*}\right): \mathrm{A}_{n}\left(F_{n^{2}}\right)_{\tilde{\Delta}}^{\mathrm{Ch}} \rightarrow \mathrm{A}_{n}(\Gamma)_{\Delta}^{\mathrm{Ch}}$ is the same morphism as in Corollary 4.5. Claim 6 implies that the images of $s(\gamma)$ by two morphisms coincide for each $\gamma \in \Gamma$. On the other hand, the fact that $\rho\left(\tilde{\alpha}_{i}\right)=\sigma\left(\alpha_{i}\right)$ implies that two morphisms send each element of $\operatorname{Im}(\phi)$ to the same image. Therefore the diagram is commutative. 
We have thus completed the proof of Lemma 5.2. Therefore we have proved Theorem 5.1.

\section{§6. Proof of the Main Theorem}

Until the previous sections we have prepared several tools for the proof of the main theorem. In the beginning of this section we prove that the action of $\mathrm{PGL}_{n}$ on $\operatorname{Rep}_{n}(\Gamma)_{\text {a.i.r. }}$ is free. Next we show that the morphism $\pi_{n, \Gamma \text {,air }}: \operatorname{Rep}_{n}(\Gamma)_{\text {a.i.r. }} \rightarrow \operatorname{Ch}_{n}^{0}(\Gamma)_{\text {a.i.r. }}$ is faithfully flat. From these results we

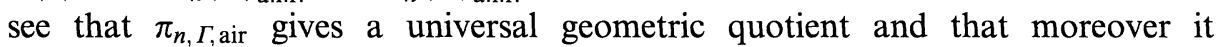
is a $\mathrm{PGL}_{n}$-principal fiber bundle. Thus we construct the character variety $\mathrm{Ch}_{n}^{0}(\Gamma)_{\text {a.i.r. }}\left(=\mathrm{Ch}_{n}(\Gamma)_{\text {a.i.r. }}\right)$.

Definition 6.1. We define the following contravariant functor:

$$
\begin{aligned}
F:(\text { Sch }) & \rightarrow(\text { Sets }) \\
X & \mapsto \operatorname{Aut}_{\mathcal{O}_{X}-\operatorname{alg}}\left(\mathbf{M}_{n}\left(\mathcal{O}_{X}\right)\right) .
\end{aligned}
$$

Note that $\mathrm{M}_{n}\left(\mathcal{O}_{X}\right)$ is a sheaf of $\mathcal{O}_{X}$-algebras on $X$.

We define the natural transformation $\xi: h_{\mathrm{PGL}_{n}} \rightarrow F$ in the following way. For a scheme $X$, each element $\sigma \in h_{\mathrm{PGL}_{n}}(X)$ is expressed by pairs $\left(U_{i}, \sigma_{i}\right)_{i \in I}$ as follows: $\left\{U_{i}\right\}_{i \in I}$ is an open covering of $X$ and $\sigma_{i} \in \operatorname{GL}_{n}\left(\mathcal{O}_{X}\left(U_{i}\right)\right)$ such that for each $x \in U_{i} \cap U_{j}$, two sections $\sigma_{i}$ and $\sigma_{j}$ coincide up to scalar multiple at a neighborhood of $x$. The maps $\operatorname{Ad}\left(\sigma_{i}\right):=\sigma_{i}^{-1} \cdot \sigma_{i}$ are automorphisms of $\mathbf{M}_{n}\left(\left.\mathcal{O}_{X}\right|_{U_{1}}\right)$ for $i \in I$ and they are glued together. Therefore we get an automorphism $\operatorname{Ad}(\sigma)$ of $\mathrm{M}_{n}\left(\mathcal{O}_{X}\right)$. This correspondence induces a natural transformation, which will be called $\xi$. We can easily check that the natural transformation $\xi$ is an isomorphism by the Skolem-Noether Theorem:

Theorem 6.2 (Skolem-Noether). Let $R$ be a local ring. For any $R$ algebra isomorphism $\sigma: \mathrm{M}_{n}(R) \rightarrow \mathrm{M}_{n}(R)$ there exists $P \in \mathrm{GL}_{n}(R)$ such that $\sigma=\operatorname{Ad}(P)\left(=P^{-1} \cdot P\right)$.

This theorem is well-known, hence we omit the proof.

Here we show that the group scheme $\mathrm{PGL}_{n}$ acts freely on the a.i.r. part. For $\alpha_{1}, \ldots, \alpha_{n^{2}} \in \Gamma$, we denote the discriminant $\Delta\left(\alpha_{1}, \ldots, \alpha_{n^{2}}\right)$ by $\Delta$. Note that $\operatorname{Rep}_{n}(\Gamma)_{\Delta}$ is the scheme representing the functor

$$
\begin{aligned}
(\text { Sch }) & \rightarrow \text { (Sets) } \\
X & \mapsto\left\{\rho \in \operatorname{Rep}_{n}(\Gamma)(X) \mid \Delta\left(\rho\left(\alpha_{1}\right), \ldots, \rho\left(\alpha_{n^{2}}\right)\right) \in \Gamma\left(X, \mathcal{O}_{X}\right)^{\times}\right\} .
\end{aligned}
$$

Then the action of $\mathrm{PGL}_{n}$ on $\operatorname{Rep}_{n}(\Gamma)_{\Delta}$ can be interpreted as 


$$
\begin{aligned}
\operatorname{Ad}_{X}: h_{\operatorname{Rep}_{n}(\Gamma)_{\Delta}}(X) \times h_{\mathrm{PGL}_{n}}(X) & \rightarrow h_{\operatorname{Rep}_{n}(\Gamma)_{\Delta}}(X) \\
(\rho, \sigma) & \mapsto \operatorname{Ad}(\sigma)(\rho) .
\end{aligned}
$$

Here we remark that $h_{\mathrm{PGL}_{n}}(X)$ can be regarded as the set of automorphisms of $\Gamma\left(X, \mathcal{O}_{X}\right)$-algebra $\mathrm{M}_{n}\left(\Gamma\left(X, \mathcal{O}_{X}\right)\right)$ through the natural transformation $\xi$. The representation $\operatorname{Ad}(\sigma)(\rho)$ for $\Gamma$ in $\operatorname{GL}_{n}\left(\Gamma\left(X, \mathcal{O}_{X}\right)\right)$ is defined by $(\operatorname{Ad}(\sigma)(\rho))(\gamma):=$ $\operatorname{Ad}(\sigma)(\rho(\gamma))$ for $\gamma \in \Gamma$.

Theorem 6.3. In the same notation as above, the morphism

$$
\begin{aligned}
\left(p_{1}, \mathrm{Ad}\right): \operatorname{Rep}_{n}(\Gamma)_{\Delta} \times_{\mathrm{Spec} \mathbf{Z}} \mathrm{PGL}_{n} \rightarrow \operatorname{Rep}_{n}(\Gamma)_{\Delta} \times_{\mathrm{Ch}_{n}^{0}(\Gamma)_{\Delta}} \operatorname{Rep}_{n}(\Gamma)_{\Delta} \\
(\rho, \sigma) \mapsto(\rho, \operatorname{Ad}(\sigma)(\rho))
\end{aligned}
$$

is an isomorphism.

Proof. It suffices to prove that for any scheme $X$ the map $\left(p_{1}, \mathrm{Ad}\right)_{X}: h_{\mathrm{Rep}_{n}(\Gamma)_{\Delta}}(X) \times h_{\mathrm{PGL}_{n}}(X) \rightarrow h_{\mathrm{Rep}_{n}(\Gamma)_{\Delta}}(X) \times_{h_{\mathrm{Ch}_{n}^{0}(\Gamma)_{\Delta}}(X)} h_{\operatorname{Rep}_{n}(\Gamma)_{\Delta}}(X) \quad$ is bijective. Throughout this proof we denote $\Gamma\left(X, \mathcal{O}_{X}\right)$ by $R$.

First we show that this map is injective. Suppose that the images of $\left(\rho_{1}, \sigma_{1}\right)$ and $\left(\rho_{2}, \sigma_{2}\right)$ by $\left(p_{1}, \mathrm{Ad}\right)_{X}$ coincide. Then it is clear that $\rho_{1}=\rho_{2}$. Put $\rho:=\rho_{1}=\rho_{2}$. The set $\{\rho(\gamma) \mid \gamma \in \Gamma\}$ generates the $R$-algebra $\mathbf{M}_{n}(R)$, since $\Delta\left(\rho\left(\alpha_{1}\right), \ldots, \rho\left(\alpha_{n^{2}}\right)\right) \in R^{\times}$. Therefore the assumption that $\operatorname{Ad}\left(\sigma_{1}\right)(\rho)=$ $\operatorname{Ad}\left(\sigma_{2}\right)(\rho)$ implies that $\operatorname{Ad}\left(\sigma_{1}\right)=\operatorname{Ad}\left(\sigma_{2}\right)$ as $R$-algebra isomorphisms of $\mathbf{M}_{n}(R)$. Hence we have $\sigma_{1}=\sigma_{2}$. Thus the injectivity has been proved.

Next for the surjectivity, it suffices to show that for any $\left(\rho, \rho^{\prime}\right) \in$ $h_{\operatorname{Rep}_{n}(\Gamma)_{\Delta}}(X) \times_{h_{\mathrm{Ch}_{n}^{0}(\Gamma)_{\Delta}}(X)} h_{\operatorname{Rep}_{n}(\Gamma)_{\Delta}}(X)$, there exists some $\sigma \in h_{\mathrm{PGL}_{n}}(X)$ such that $\operatorname{Ad}(\sigma)(\rho)=\rho^{\prime}$. Note that the discriminants $\Delta\left(\rho\left(\alpha_{1}\right), \rho\left(\alpha_{2}\right), \ldots, \rho\left(\alpha_{n^{2}}\right)\right)$ and $\Delta\left(\rho^{\prime}\left(\alpha_{1}\right), \rho^{\prime}\left(\alpha_{2}\right), \ldots, \rho^{\prime}\left(\alpha_{n^{2}}\right)\right)$ are invertible elements of $R$. Hence the sets $\left\{\rho\left(\alpha_{1}\right), \ldots, \rho\left(\alpha_{n^{2}}\right)\right\}$ and $\left\{\rho^{\prime}\left(\alpha_{1}\right), \ldots, \rho^{\prime}\left(\alpha_{n^{2}}\right)\right\}$ are $R$-bases of $\mathbf{M}_{n}(R)$. Now we define the $R$-linear map $\phi$ by

$$
\begin{aligned}
\phi: \mathrm{M}_{n}(R) & \rightarrow \mathrm{M}_{n}(R) \\
\rho\left(\alpha_{i}\right) & \mapsto \rho^{\prime}\left(\alpha_{i}\right) .
\end{aligned}
$$

We claim that $\phi$ is an $R$-algebra isomorphism. Suppose that $\psi, \psi^{\prime}$ : $\mathrm{A}_{n}(\Gamma)_{\Delta} \rightarrow R$ are the ring homomorphisms corresponding to $\rho$ and $\rho^{\prime}$, respectively. Then we get the commutative diagram:

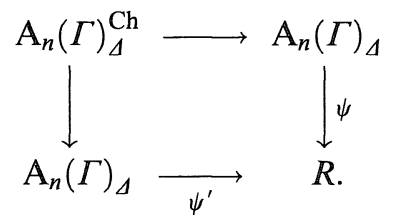


Denote by $\chi: \mathrm{A}_{n}(\Gamma)_{\Delta}^{\mathrm{Ch}} \rightarrow R$ the ring homomorphism determined from the diagram. By Theorem 4.1 we obtain the following equality:

$$
\rho(\gamma)=\left(\rho\left(\alpha_{1}\right), \ldots, \rho\left(\alpha_{n^{2}}\right)\right) T\left(\begin{array}{c}
\chi\left(s\left(\alpha_{1} \gamma\right)\right) \\
\vdots \\
\chi\left(s\left(\alpha_{n^{2}} \gamma\right)\right)
\end{array}\right) \quad \text { for each } \gamma \in \Gamma
$$

where

$$
T=\left(\begin{array}{ccc}
\chi\left(s\left(\alpha_{1} \alpha_{1}\right)\right) & \cdots & \chi\left(s\left(\alpha_{1} \alpha_{n^{2}}\right)\right) \\
\vdots & \chi\left(s\left(\alpha_{i} \alpha_{j}\right)\right) & \vdots \\
\chi\left(s\left(\alpha_{n^{2}} \alpha_{1}\right)\right) & \cdots & \chi\left(s\left(\alpha_{n^{2}} \alpha_{n^{2}}\right)\right)
\end{array}\right)^{-1} \in \mathrm{GL}_{n^{2}}(R)
$$

The similar equation also holds for $\rho^{\prime}$. Hence $\phi(\rho(\gamma))=\rho^{\prime}(\gamma)$ for each $\gamma \in \Gamma$. In particular we obtain

$$
\begin{aligned}
\phi\left(\rho\left(\alpha_{i} \alpha_{j}\right)\right) & =\rho^{\prime}\left(\alpha_{i} \alpha_{j}\right) \\
& =\rho^{\prime}\left(\alpha_{i}\right) \rho^{\prime}\left(\alpha_{j}\right) \\
& =\phi\left(\rho\left(\alpha_{i}\right)\right) \phi\left(\rho\left(\alpha_{j}\right)\right) .
\end{aligned}
$$

Therefore the linear map $\phi$ is an $R$-algebra isomorphism. For the $R$-algebra isomorphism $\phi: \mathrm{M}_{n}(R) \rightarrow \mathrm{M}_{n}(R)$ there exists $\sigma \in h_{\mathrm{PGL}_{n}}(X)$ such that $\operatorname{Ad}(\sigma)=\phi$. In particular $\operatorname{Ad}(\sigma)(\rho)=\rho^{\prime}$. This implies the surjectivity.

We have thus proved the theorem.

The previous theorem can be extended to the next statement.

Corollary 6.4. In the same notation as above, the morphism

$$
\operatorname{Rep}_{n}(\Gamma)_{\text {a.i.r. }} \times{ }_{\operatorname{Spec} \mathbf{Z}} \operatorname{PGL}_{n} \stackrel{\left(p_{1}, \operatorname{Ad}\right)}{\longrightarrow} \operatorname{Rep}_{n}(\Gamma)_{\text {a.i.r. }} \times_{\mathrm{Ch}_{n}^{0}(\Gamma)_{\mathrm{a} 1 \mathrm{r}}} \operatorname{Rep}_{n}(\Gamma)_{\text {a.i.r. }}
$$

is an isomorphism.

In particular we get the following corollary.

Corollary 6.5. The action of $\mathrm{PGL}_{n}$ on $\operatorname{Rep}_{n}(\Gamma)_{\text {a.i.r. }}$ is free. In other words, the following morphism is a closed immersion:

$$
\left(p_{1}, \operatorname{Ad}\right): \operatorname{Rep}_{n}(\Gamma)_{\text {a.i.r. }} \times_{\operatorname{Spec} \mathbf{Z}} \operatorname{PGL}_{n} \rightarrow \operatorname{Rep}_{n}(\Gamma)_{\text {a.i.r. }} \times_{\operatorname{Spec} \mathbf{Z}} \operatorname{Rep}_{n}(\Gamma)_{\text {a.i.r. }} .
$$

Remark 6.6. Corollaries 6.4 and 6.5 tell us that any absolutely irreducible representation is a properly stable point in $\operatorname{Rep}_{n}(\Gamma)$ by $\operatorname{PGL}_{n}$ with respect to the canonical linearization on the trivial line bundle in the sense of GIT [2]. Here we show the converse: if $\rho$ is not absolutely irreducible, then $\rho$ is not properly stable. If $x: \operatorname{Spec} \Omega \rightarrow \operatorname{Rep}_{n}(\Gamma)$ is a geometric point in 
$\operatorname{Rep}_{n}(\Gamma)$ and if the corresponding representation $\rho: \Gamma \rightarrow \mathrm{GL}_{n}(\Omega)$ is not absolutely irreducible, then $\rho$ has a non-trivial invariant subspace. With a suitable basis, we can write

$$
\rho(\gamma)=\left(\begin{array}{cc}
\rho_{1}(\gamma) & \rho^{\prime}(\gamma) \\
0 & \rho_{2}(\gamma)
\end{array}\right)
$$

If the stabilizer of $x$ has dimension $\geq 1$, then $x$ is not properly stable. Suppose that the stabilizer of $x$ has dimension 0 . Consider the map

$$
\mathbb{G}_{m} \ni t \mapsto\left(\begin{array}{cc}
t^{2} \mathbf{I}_{n_{1}} & 0 \\
0 & t \mathbf{I}_{n_{2}}
\end{array}\right)\left(\begin{array}{cc}
\rho_{1}(\gamma) & \rho^{\prime}(\gamma) \\
0 & \rho_{2}(\gamma)
\end{array}\right)\left(\begin{array}{cc}
t^{-2} \mathbf{I}_{n_{1}} & 0 \\
0 & t^{-1} \mathbb{I}_{n_{2}}
\end{array}\right) .
$$

We see that it tends to

$$
\left(\begin{array}{cc}
\rho_{1}(\gamma) & 0 \\
0 & \rho_{2}(\gamma)
\end{array}\right)
$$

as $t \rightarrow 0$. Since the closure of the orbit of $\rho$ contains an another orbit, the orbit of $\rho$ by $\mathrm{PGL}_{n}$ is not closed. Hence $x$ is not properly stable.

Therefore $x \in \operatorname{Rep}_{n}(\Gamma)$ is properly stable if and only if $x$ is a.i.r.

For proving the existence of the quotient, we show that the morphism $\pi_{n, \Gamma, \Delta}: \operatorname{Rep}_{n}(\Gamma)_{\Delta} \rightarrow \mathrm{Ch}_{n}^{0}(\Gamma)_{\Delta}$ is faithfully flat.

Theorem 6.7. The morphism $\operatorname{Rep}_{n}(\Gamma)_{\Delta} \rightarrow \mathrm{Ch}_{n}^{0}(\Gamma)_{\Delta}$ is faithfully flat.

Proof. By Theorem 5.1, we only have to prove that the "prototype" $\pi_{n, \mathrm{~F}_{n^{2}}, \tilde{\Delta}}: \operatorname{Rep}_{n}\left(\mathrm{~F}_{n^{2}}\right)_{\tilde{\Delta}} \rightarrow \mathrm{Ch}_{n}^{0}\left(\mathrm{~F}_{n^{2}}\right)_{\tilde{\Delta}}$ is faithfully flat. We denote $\pi_{n, \mathrm{~F}_{n^{2}}, \tilde{\Delta}}$ by $\phi$. First we prove that $\phi$ is surjective. It suffices to prove that the morphism $\phi_{\Omega}$ : $\operatorname{Rep}_{n}\left(\mathrm{~F}_{n^{2}}\right)_{\bar{\Delta}} \otimes_{\mathbf{Z}} \Omega \rightarrow \mathrm{Ch}_{n}^{0}\left(\mathrm{~F}_{n^{2}}\right)_{\bar{\Delta}} \otimes_{\mathbf{Z}} \Omega$ is surjective for any algebraically closed field $\Omega$. From Donkin's Theorem (Theorem 2.12) we get an isomorphism $\left(\mathrm{A}_{n}\left(\mathrm{~F}_{n^{2}}\right) \otimes_{\mathrm{Z}} \Omega\right)_{\tilde{\Delta}}^{\mathrm{PGL}_{n}} \cong \mathrm{A}_{n}\left(\mathrm{~F}_{n^{2}}\right)_{\tilde{\Delta}}^{\mathrm{PGL}_{n}} \otimes_{\mathrm{Z}} \Omega$. Hence $\phi_{\Omega}: \operatorname{Rep}_{n}\left(\mathrm{~F}_{n^{2}}\right)_{\tilde{\Delta}} \otimes_{\mathrm{Z}} \Omega \rightarrow$ $\mathrm{Ch}_{n}^{0}\left(\mathrm{~F}_{n^{2}}\right)_{\tilde{\Delta}} \otimes_{\mathrm{Z}} \Omega$ is a categorical quotient. From Corollary 6.5 the action of $\mathrm{PGL}_{n}$ is free, so the quotient map gives a geometric quotient. In particular the quotient map is surjective. This completes the first step.

Secondly we prove that the morphism $\phi$ is flat. We recall that $\operatorname{Rep}_{n}\left(\mathbb{F}_{n^{2}}\right)_{\bar{\Delta}}$ is smooth over $\mathbb{Z}$ from Example 2.6. Since $\operatorname{Rep}_{n}\left(\mathrm{~F}_{n^{2}}\right)_{\bar{\Delta}} \otimes_{\mathbf{Z}} \Omega \rightarrow \mathrm{Ch}_{n}^{0}\left(\mathrm{~F}_{n^{2}}\right)_{\bar{\Delta}} \otimes_{\mathbf{Z}} \Omega$ is a geometric quotient and the action is free, $\mathrm{Ch}_{n}^{0}\left(\mathrm{~F}_{n^{2}}\right)_{\bar{\Delta}} \otimes_{\mathbb{Z}} \Omega$ is nonsingular for any algebraically closed field $\Omega$. Therefore $\mathrm{Ch}_{n}^{0}\left(\mathrm{~F}_{n^{2}}\right)_{\tilde{\Delta}}$ is smooth over $\mathbb{Z}$. In particular $\mathrm{Ch}_{n}^{0}\left(\mathrm{~F}_{n^{2}}\right)_{\tilde{\Delta}}$ is a regular scheme. Any fiber of the morphism $\phi$ is isomorphic to $\mathrm{PGL}_{n}$, so the dimensions of fibers are constant. This implies that the morphism $\phi$ is flat.

We have thus completed the proof.

We have come to the final stage of the proof of the main theorem. 


\section{Proof of Theorem 1.3.}

First we prove that the morphism $\pi_{n, \Gamma \text {,air }}: \operatorname{Rep}_{n}(\Gamma)_{\text {a.i.r. }} \rightarrow \operatorname{Ch}_{n}^{0}(\Gamma)_{\text {a.i.r. }}$ gives a universal geometric quotient by $\mathrm{PGL}_{n}$. By Corollary 2.13 and Theorem 6.3 and Theorem 6.7, we see that the prototype $\pi_{n, \mathrm{~F}_{n^{2}}, \tilde{\Delta}}: \operatorname{Rep}_{n}\left(\mathrm{~F}_{n^{2}}\right)_{\tilde{\Delta}} \rightarrow \mathrm{Ch}_{n}^{0}\left(\mathrm{~F}_{n^{2}}\right)_{\tilde{\Delta}}$ gives a universal geometric quotient. Theorem 5.1 follows that the morphism $\pi_{n, \Gamma \text {, air }}$ is a universal geometric quotient for an arbitrary group $\Gamma$.

Next we show that $\operatorname{Ch}_{n}^{0}(\Gamma)_{\text {a.i.r. }}$ is a coarse moduli scheme associated to $\mathscr{E} q \mathscr{A} \mathscr{I} \mathscr{R}_{n}(\Gamma)$. For an element $[\rho] \in \mathscr{E} q \mathscr{A} \mathscr{I} \mathscr{R}_{n}(\Gamma)(X)$ with a scheme $X$, pick a representative $\rho: \Gamma \rightarrow \mathrm{GL}_{n}\left(\Gamma\left(X, \mathcal{O}_{X}\right)\right)$ of $[\rho]$. Consider the composite of the morphism $X \rightarrow \operatorname{Rep}_{n}(\Gamma)_{\text {a.i.r. }}$ associated to $\rho$ and the quotient morphism $\pi_{n, \Gamma \text {, air }}$ : $\operatorname{Rep}_{n}(\Gamma)_{\text {a.i.r. }} \rightarrow \mathrm{Ch}_{n}^{0}(\Gamma)_{\text {a.i.r. }}$. Then we obtain an element of $h_{\mathrm{Ch}_{n}^{0}(\Gamma)_{\mathrm{a} 1 \Gamma}}(X)$. This correspondence induces a natural transformation $\tau: \mathscr{E} q \mathscr{A} \mathscr{I} \mathscr{R}_{n}(\Gamma) \stackrel{\text { a }}{\rightarrow} h_{\mathrm{Ch}_{n}^{0}(\Gamma)_{\mathrm{a} 1 \mathrm{r}}}$. Since $\operatorname{Ch}_{n}^{0}(\Gamma)_{\text {a.i.r. }}$ is a geometric quotient of $\operatorname{Rep}_{n}(\Gamma)_{\text {a.i.r. }}$, we can easily see that the natural transformation $\tau$ satisfies (i) and (ii) of Theorem 1.3. The separatedness of $\mathrm{Ch}_{n}^{0}(\Gamma)_{\text {a.i.r. }}$ follows from that it is an open subscheme of the affine scheme $\mathrm{Ch}_{n}^{0}(\Gamma)$.

From the proof of Theorem 1.3 we obtain the next corollary.

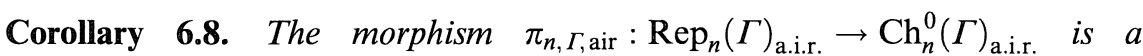
universal geometric quotient by $\mathrm{PGL}_{n}$. Moreover it is a $\mathrm{PGL}_{n}$-principal fiber bundle.

Remark 6.9. From Corollary 6.8 we have $\mathrm{Ch}_{n}(\Gamma)_{\text {a.i.r. }}=\mathrm{Ch}_{n}^{0}(\Gamma)_{\text {a.i.r. }}$. Therefore we have $\mathrm{A}_{n}(\Gamma)_{\Delta}^{\mathrm{Ch}}=\mathrm{A}_{n}(\Gamma)_{\Delta}^{\mathrm{PGL}_{n}}$. Furthermore $\mathrm{A}_{n}(\Gamma)_{\Delta}^{\mathrm{Ch}}$ is the universal invariant subring of $\mathrm{A}_{n}(\Gamma)_{\Delta}$ by $\mathrm{PGL}_{n}$.

Remark that if $\Gamma$ is a finitely generated group, then $\mathrm{Ch}_{n}(\Gamma)_{\text {a.i.r. }}$ is separated of finite type over $\mathbf{Z}$ (we see that $\mathrm{Ch}_{n}(\Gamma)_{\text {a.i.r. }}$ is quasi-compact because it is the

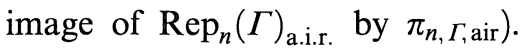

Here we name the universal geometric quotient of the a.i.r. part by $\mathrm{PGL}_{n}$, or the coarse moduli scheme of equivalence classes of a.i.r.

Definition 6.10 (Definition of the character variety). The scheme $\mathrm{Ch}_{n}(\Gamma)_{\text {a.i.r. }}$ is called the (a.i.r.) character variety of degree $n$ for $\Gamma$.

Remark 6.11. Suppose that $\Gamma$ is a finitely generated group. In general theory we see that the morphism $\operatorname{Rep}_{n}(\Gamma) \rightarrow \mathrm{Ch}_{n}(\Gamma)$ is a uniform categorical quotient (in particular the scheme $\mathrm{Ch}_{n}(\Gamma)$ is of finite type over $\mathbf{Z}$ ). For example, see [2] or [11]. We also call the scheme $\mathrm{Ch}_{n}(\Gamma)$ the character variety of degree $n$ for $\Gamma$.

Corollary 6.8 implies that two absolutely irreducible representations $\rho, \rho^{\prime}: \Gamma \rightarrow \mathrm{GL}_{n}(R)$ are equivalent if and only if $c_{j}(\rho(\gamma))=c_{j}\left(\rho^{\prime}(\gamma)\right)$ for $1 \leq j \leq n$ and for each $\gamma \in \Gamma$. Moreover we obtain the next theorem. 
Theorem 6.12. Let $R$ be a commutative ring and $\Gamma$ a group. Let $\rho, \rho^{\prime}: \Gamma \rightarrow \mathrm{GL}_{n}(R)$ be two representations. Suppose that $\rho$ is absolutely irreducible. Then

$$
\rho \sim \rho^{\prime} \Leftrightarrow \operatorname{tr}(\rho(\gamma))=\operatorname{tr}\left(\rho^{\prime}(\gamma)\right) \quad \text { for each } \gamma \in \Gamma .
$$

In particular, $\rho^{\prime}$ is also absolutely irreducible under these equivalent conditions.

Proof. Suppose that $\rho$ and $\rho^{\prime}$ are equivalent. Then for each prime ideal $\wp \in \operatorname{Spec} R$, we have $P^{-1} \rho P=\rho^{\prime}$ for a suitable matrix $P \in \mathrm{GL}_{n}$ on a neighborhood of $\wp$. Locally we obtain $\operatorname{tr}(\rho(\gamma))=\operatorname{tr}\left(\rho^{\prime}(\gamma)\right)$ for each $\gamma \in \Gamma$. Hence the equalities hold globally.

Suppose that $\operatorname{tr}(\rho(\gamma))=\operatorname{tr}\left(\rho^{\prime}(\gamma)\right)$ for each $\gamma \in \Gamma$. The absolute irreducibility of $\rho$ implies that of $\rho^{\prime}$ since for any $\alpha_{1}, \ldots, \alpha_{n^{2}} \in \Gamma$ we have $\operatorname{det}\left(\operatorname{tr}\left(\rho\left(\alpha_{i} \alpha_{j}\right)\right)\right)=$ $\operatorname{det}\left(\operatorname{tr}\left(\rho^{\prime}\left(\alpha_{i} \alpha_{j}\right)\right)\right) \quad(c f$. Remark 3.2). First we prove the special case: if $\operatorname{det}\left(\operatorname{tr}\left(\rho\left(\alpha_{i} \alpha_{j}\right)\right)\right)=\operatorname{det}\left(\operatorname{tr}\left(\rho^{\prime}\left(\alpha_{i} \alpha_{j}\right)\right)\right) \in R^{\times}$for some $\alpha_{1}, \alpha_{2}, \ldots, \alpha_{n^{2}} \in \Gamma$, then there exists a unique $R$-algebra isomorphism $\phi: \mathrm{M}_{n}(R) \rightarrow \mathrm{M}_{n}(R)$ such that $\phi(\rho(\gamma))=\rho^{\prime}(\gamma)$ for each $\gamma \in \Gamma$. Note that the sets $\left\{\rho\left(\alpha_{1}\right), \ldots, \rho\left(\alpha_{n^{2}}\right)\right\}$ and $\left\{\rho^{\prime}\left(\alpha_{1}\right), \ldots, \rho^{\prime}\left(\alpha_{n^{2}}\right)\right\}$ are $R$-bases of $\mathbf{M}_{n}(R)$ over $R$. We define the $R$-linear map $\phi: \mathrm{M}_{n}(R) \rightarrow \mathrm{M}_{n}(R)$ by $\phi\left(\rho\left(\alpha_{i}\right)\right)=\rho^{\prime}\left(\alpha_{i}\right)$ for $i=1,2, \ldots, n^{2}$. As in the proof of Theorem 6.3, we can prove that $\phi$ is an $R$-algebra isomorphism and that $\phi(\rho(\gamma))=\rho^{\prime}(\gamma)$ for each $\gamma \in \Gamma$. We easily see the uniqueness of $\phi$.

Next we prove the general case. Since $\rho$ and $\rho^{\prime}$ are absolutely irreducible, there exist an affine open covering $\operatorname{Spec} R=\bigcup_{i=1}^{N} U_{i}$ and suitable elements $\alpha_{1}^{(i)}, \ldots, \alpha_{n^{2}}^{(i)} \in \Gamma$ for $i=1, \ldots, N$ such that the discriminants $\Delta\left(\rho\left(\alpha_{1}^{(i)}\right), \ldots, \rho\left(\alpha_{n^{2}}^{(i)}\right)\right)$ and $\Delta\left(\rho^{\prime}\left(\alpha_{1}^{(i)}\right), \ldots, \rho^{\prime}\left(\alpha_{n^{2}}^{(i)}\right)\right)$ are invertible on each $U_{i}$. From the special case we obtain unique $R_{i}$-algebra isomorphisms $\phi_{i}: \mathrm{M}_{n}\left(R_{i}\right) \rightarrow \mathrm{M}_{n}\left(R_{i}\right)$ such that $\phi_{i}(\rho(\gamma))=\rho^{\prime}(\gamma)$ for each $\gamma \in \Gamma$. Here we denote $\Gamma\left(U_{i}, \mathcal{O}_{U_{l}}\right)$ by $R_{i}$. From the uniqueness we see that $\left\{\phi_{i}\right\}$ are glued together. Hence we have the desired $R$-algebra isomorphism $\phi$. This completes the proof.

The following theorem can be proved as above.

Theorem 6.13. Let $A$ be an associative algebra over a commutative ring $R$. Let $S$ be a commutative R-algebra, and let $\rho, \rho^{\prime}: A \rightarrow \mathrm{M}_{n}(S)$ be R-algebra homomorphisms. Suppose that $\rho$ is absolutely irreducible. Then

$$
\rho \sim \rho^{\prime} \Leftrightarrow \operatorname{tr}(\rho(a))=\operatorname{tr}\left(\rho^{\prime}(a)\right) \quad \text { for each } a \in A .
$$

In particular, $\rho^{\prime}$ is also absolutely irreducible under these equivalent conditions.

The statement in Theorem 6.13 has been proved in [1] and [10] for the case that $R$ is a local ring and that $S=R$. Theorem 6.12 and Theorem 6.13 are generalization of these results. ${ }^{1}$

1 The author was informed about these papers by the referees. The author improved the statement of Theorem 6.12 by helpful suggestions of the referees, although the statement in Theorem 6.12 was that $\rho \sim \rho^{\prime}$ if and only if $c_{J}(\rho(\gamma))=c_{J}\left(\rho^{\prime}(\gamma)\right)$ in the first version. 
Remark 6.14. In Theorems 6.12 and 6.13 we can not avoid the assumption that $\rho$ is absolutely irreducible. Indeed, let us define $\rho, \rho^{\prime}: \mathbb{Z} \rightarrow$ $\mathrm{GL}_{2}(R)$ by

$$
\rho(1):=\left(\begin{array}{ll}
1 & 0 \\
0 & 1
\end{array}\right), \quad \rho^{\prime}(1):=\left(\begin{array}{ll}
1 & 1 \\
0 & 1
\end{array}\right)
$$

Then we see that $\rho$ and $\rho^{\prime}$ are not equivalent, however $\operatorname{tr}(\rho(m))=\operatorname{tr}\left(\rho^{\prime}(m)\right)$ and $\operatorname{det}(\rho(m))=\operatorname{det}\left(\rho^{\prime}(m)\right)$ for each $m \in \mathbf{Z}$.

Remark 6.15. We say that the Skolem-Noether Theorem holds in a commutative ring $R$ if there exists $P \in \mathrm{GL}_{n}(R)$ such that $\sigma=\operatorname{Ad}(P)$ for an arbitrary $R$-algebra isomorphism $\sigma: \mathrm{M}_{n}(R) \rightarrow \mathrm{M}_{n}(R)$. If the Skolem-Noether Theorem holds in $R$, then $\rho$ and $\rho^{\prime}$ are equivalent if and only if there exists $P \in \mathrm{GL}_{n}(R)$ such that $\operatorname{Ad}(P)(\rho)=\rho^{\prime}$.

Here let us consider a sufficient condition that the Skolem-Noether Theorem holds. We can regard the group scheme $\mathrm{PGL}_{n}$ as the open subscheme $\left\{\operatorname{det}\left(X_{i j}\right) \neq 0\right\}$ of the projective space $\mathbf{P}^{n^{2}-1}=\operatorname{Proj} \mathbf{Z}\left[X_{i j} \mid 1 \leq i, j \leq n\right]$. Hence for a scheme $X$ we get

$$
\begin{aligned}
& h_{\mathrm{PGL}_{n}}(X) \\
& =\left\{\begin{array}{l|l}
\left(\mathscr{L},\left\{s_{i j}\right\}_{1 \leq i, j \leq n}\right) & \begin{array}{l}
\mathscr{L} \in \operatorname{Pic}(X), s_{i j} \in \Gamma(X, \mathscr{L}) \\
\text { such that the section } \operatorname{det}\left(s_{i j}\right)_{1 \leq i, j \leq n} \\
\text { of } \mathscr{L}^{\otimes n} \text { nowhere vanishes }
\end{array}
\end{array}\right\} \sim \sim
\end{aligned}
$$

Here we denote by $\operatorname{Pic}(X)$ the Picard group of $X$, that is, the group of isomorphism classes of line bundles on $X$. We say that $\left(\mathscr{L},\left\{s_{i j}\right\}\right) \sim\left(\mathscr{L}^{\prime},\left\{s_{i j}^{\prime}\right\}\right)$ if the corresponding morphisms from $X$ to $\mathbf{P}^{n^{2}-1}$ coincide. If $\left(\mathscr{L},\left\{s_{i j}\right\}_{1 \leq i, j \leq n}\right) \in$ $h_{\mathrm{PGL}_{n}}(X)$, then the line bundle $\mathscr{L}^{\otimes n}$ is trivial. In particular if the Picard group $\operatorname{Pic}(X)$ is a torsion free group, then $\mathscr{L}$ is trivial and $\left(s_{i j}\right)_{1 \leq i, j \leq n} \in$ $\mathrm{GL}_{n}\left(\Gamma\left(X, \mathcal{O}_{X}\right)\right)$. By the isomorphism $\xi_{X}: h_{\mathrm{PGL}_{n}}(X) \stackrel{\sim}{\longrightarrow} \operatorname{Aut}_{\mathfrak{C}_{X}-\text { alg }} \mathrm{M}_{n}\left(\mathcal{O}_{X}\right)$, we see that the Skolem-Noether Theorem holds in a commutative ring $R$ if the Picard group $\operatorname{Pic}(\operatorname{Spec} R)$ is a torsion free group. For example, if $R$ is a UFD, then the Picard group is trivial and the Skolem-Noether Theorem holds.

We end this paper by introducing the representation variety and the character variety in the $\mathrm{SL}_{n}$ case. The similar statements hold for the $\mathrm{SL}_{n}$ case.

Definition 6.16 (The $\mathrm{SL}_{n}$ case). Let $J$ denote the ideal of $\mathrm{A}_{n}(\Gamma)^{\mathrm{Ch}}$ generated by $\{\mathrm{d}(\gamma)-1 \mid \gamma \in \Gamma\}$. Here we $\operatorname{denote} \operatorname{det}\left(\sigma_{\Gamma}(\gamma)\right)$ by $d(\gamma)$. We set

$$
\begin{aligned}
\overline{\mathrm{A}}_{n}^{\mathrm{Ch}}(\Gamma) & :=\mathrm{A}_{n}(\Gamma)^{\mathrm{Ch}} / J, \\
\overline{\mathrm{A}}_{n}(\Gamma) & :=\mathrm{A}_{n}(\Gamma) / J \cdot \mathrm{A}_{n}(\Gamma) .
\end{aligned}
$$


We call

$$
\begin{aligned}
\overline{\mathrm{Ch}}_{n}(\Gamma) & :=\operatorname{Spec} \overline{\mathrm{A}}_{n}^{\mathrm{Ch}}(\Gamma), \\
\overline{\operatorname{Rep}}_{n}(\Gamma) & :=\operatorname{Spec} \overline{\mathrm{A}}_{n}(\Gamma)
\end{aligned}
$$

the character variety and the representation variety of degree $n$ for $\Gamma$ in $\mathrm{SL}_{n}$, respectively. The a.i.r. parts $\overline{\mathrm{Ch}}_{n}(\Gamma)_{\text {a.i.r. }}$ and $\overline{\operatorname{Rep}}_{n}(\Gamma)_{\text {a.i.r. }}$ are defined in the similar way as the $\mathrm{GL}_{n}$ case.

Remark 6.17. The scheme $\overline{\operatorname{Rep}}_{n}(\Gamma)$ is the closed subscheme of $\operatorname{Rep}_{n}(\Gamma)$ consisting of representations in $\mathrm{SL}_{n}$; the scheme $\overline{\operatorname{Rep}}_{n}(\Gamma)_{\text {a.i.r. }}$ is the closed subscheme of $\operatorname{Rep}_{n}(\Gamma)_{\text {a.i.r. }}$ consisting of absolutely irreducible representations in $\mathrm{SL}_{n}$. The group scheme $\operatorname{PGL}_{n}$ acts on $\overline{\operatorname{Rep}}_{n}(\Gamma)$ and $\overline{\operatorname{Rep}}_{n}(\Gamma)_{\text {a.i.r. }}$ by $\rho \mapsto P^{-1} \rho P$, where $P \in \operatorname{PGL}_{n}$ and $\rho \in \overline{\operatorname{Rep}}_{n}(\Gamma)$ or $\overline{\operatorname{Rep}}_{n}(\Gamma)_{\text {a.i.r. }}$. The schemes $\overline{\mathrm{Ch}}_{n}(\Gamma)$ and $\overline{\mathrm{Ch}}_{n}(\Gamma)_{\text {a.i.r. }}$ are closed subschemes of $\mathrm{Ch}_{n}(\Gamma)$ and $\mathrm{Ch}_{n}(\Gamma)_{\text {a.i.r. }}$, respectively. There are natural morphisms $\overline{\operatorname{Rep}}_{n}(\Gamma) \rightarrow \overline{\mathrm{Ch}}_{n}(\Gamma)$ and $\overline{\operatorname{Rep}}_{n}(\Gamma)_{\text {a.i.r. }} \rightarrow \overline{\mathrm{Ch}}_{n}(\Gamma)_{\text {a.i.r. }}$.

Theorem 6.18. The morphism $\overline{\operatorname{Rep}}_{n}(\Gamma)_{\text {a.i.r. }} \rightarrow \overline{\mathrm{Ch}}_{n}(\Gamma)_{\text {a.i.r. }}$ is a universal geometric quotient by the adjoint action of $\mathrm{PGL}_{n}$. Moreover the quotient morphism is a $\mathrm{PGL}_{n}$-principal fiber bundle. In particular $\overline{\mathrm{Ch}}_{n}(\Gamma)_{\text {a.i.r. }}$ is the coarse moduli scheme for the $\mathrm{SL}_{n}$ case.

Proof. The morphism is obtained by the base change of the quotient morphism in the $\mathrm{GL}_{n}$ case. The statement follows from the main theorem.

\section{References}

[1] Carayol, H., Formes modulaires et représentations galoisiennes à valeurs dans un anneau local complet, Comtemp. Math., 165 (1994), 213-237.

[2] Mumford, D., Fogarty, J. and Kirwan, F., Geometric invariant theory, third edition, Springer-Verlag, 1994.

[ 3 ] Donkin, S., Invariants of several matrices, Invent. Math., 110 (1992), 389-401.

[4] Jantzen, J. C., Representation of algebraic groups, Academic Press, Inc., 1987.

[5] King, A. D., Moduli of representations of finite dimensional algebras, Quart. J. Math. Oxford Ser. (2), 45 (1994), 515-530.

[6] Milne, J. S., Étale cohomology, Princeton University Press, Princeton, 1980.

[7] Nakamoto, K., On monads and vector bundles over the projective plane, Preprint, KyotoMath 2000-02, 2000.

[ 8 ] - On the Structure of the Character Rings, Master's thesis, Kyoto University, 1995, (in Japanese).

[9] Saito, K., Representation Varieties of a Finitely Generated Group into $S L_{2}$ or GL 2 , Preprint RIMS-958, 1993. 
[10] Serre, J.-P., Représentaions linéaires sur des anneaux locaux, d'après carayol, Publ. Inst. Math. Jussieu, (1995), no. 49.

[11] Seshadri, C. S., Geometric Reductivity over arbitrary case, Adv. Math., 26 (1977), 225-274.

[12] Tits, J., Free subgroups in linear groups, J. Algebra, 20 (1972), 250-270. 
